\title{
Conformal Einstein evolution *
}

\author{
Helmut Friedrich \\ Max-Planck-Institut für Gravitationsphysik \\ Am Mühlenberg 1 \\ 14476 Golm \\ Germany
}

October 24, 2018

\begin{abstract}
We discuss various properties of the conformal field equations and their consequences for the asymptotic structure of space-times.
\end{abstract}

\section{Introduction}

The causal structure, which decides whether a signal can be sent between two space-time points, is of fundamental importance for all of field theory. In the case of Einstein's theory of gravitation, general relativity, it acquires a particular significance, because the null cone structure underlying the causal relations is itself a basic unknown of the field equations and as such subject to the causal relations in the small.

From the beginning of general relativity the effect of 'deflection of light in gravitational fields', demonstrated nowadays so clearly by the gravitational lensing phenomena, has been considered theoretically and observationally as one of the clearest manifestations of the deviation of the light cone field in general relativity from that of special relativity. As seen in the article by G. Galloway in this volume, which shows, among other things, the ubiquity of caustics on wave fronts in solutions to Einstein's field equations, it is not only a local effect but also related to the global structure of space-times.

This relation to the global structure of space-time becomes quite pronounced in the case of black holes, which seem to be on the verge now of also becoming 'observed' phenomena. The observational situation as well as various theoretical aspects of the idea are addressed directly in P. Chruściel's article in this volume. However, tacitly or explicitly, the related questions of gravitational collapse, merger of black holes, generation of gravitational radiation, singularities of gravitational fields, etc. motivate to a large extent all studies in this volume.

* To appear in: J. Frauendiener, H. Friedrich (eds.), "The Conformal Structure of Spacetime: Geometry, Analysis, Numerics", Springer, Lecture Notes in Physics, Berlin, 2002. 
The dual and critical role of the light cone structure in Einstein's theory and in physics in general led some researchers to speculate that the null cone structure might be a classical space-time reflection of a fundamental feature of a quantum theory of gravitation. This motivated efforts to recast the classical theory into forms which put the null cone structure, or associated structures like wave fronts, into the foreground. The article by S. Frittelli, N. Kamran, and E. Newman as well as the article by R. Penrose in this volume are aiming into this direction. Trying instead to derive qualitative and quantitative results about the solutions, the remaining articles explore and exploit, in one way or other, the light cone structure along more conventional lines.

That in studies of the global structure of gravitational fields the light cone structure acquires a particular technical interest, has been demonstrated most impressively by the proofs of the singularity theorems ([51], [67], cf. also [52]). It provides the most important tool for analyzing space-times in the large. While these theorems show that under reasonable assumptions space-times are singular in the sense that they contain non-complete causal geodesics, they do not provide any information on the specific nature of the singularities. It is expected that in general curvature singularities will arise, however, the theorems give not the slightest hint whether this will be the case, not to mention the causal nature of the singularities or their strength.

In spite of various attempts to obtain more detailed results, the related problem of cosmic censorship (cf. [72, [71], [79]) still poses some of the most important outstanding questions of classical general relativity. While the limited use of the field equations made in the singularity theorems contributes to their generality, the same fact is responsible for the scarcity of details supplied by them. In view of the enormous richness of pathological behaviour observed on the level of pure space-time theory (cf. [22] and the literature given there) it is not surprising that attacks at the problem of cosmic censorship which rely mainly on the topological methods used in the proof of the singularity theorems met little success.

To disclose the more subtle secrets of Einstein's theory we need a deeper understanding of the causal structure in the context of Einstein's field equations. In any serious investigation of the problem the consequences of these equations, which represent the principal restrictive principle of the theory, must be taken into account in all completeness. Getting control on the evolution process defined by Einstein's equations still remains the most important technical task in classical general relativity.

There arises the question of how to combine the analysis of the field equations with that of the causal structure. In Einstein's theory these structures are particularly closely related. By the local causality requirement the causal structure is determined by the null cones of the metric. Conversely, provided certain pathologies are excluded, the causal structure can be used to reconstruct the null cone structure. Moreover, the physical characteristics of the field equations, which largely govern the evolution process, are given by the null hypersurfaces defined by the solutions. These tight relations between causal structure, null cone structure, and characteristics structure clearly offer possibilities for the 
desired analysis, but it may be noted that they are also responsible for the quasi-linearity of the equations which, together with their gauge hyperbolicity, is the main source of our difficulties.

The null cone structure is equivalent to the conformal structure, given by the information which is retained if the metric is subject to arbitrary conformal rescalings $g \rightarrow \Omega^{2} g$ with conformal factors $\Omega>0$. This may allow us to relate the null cone structure in a new way to the structure of the field equations and to the large scale behaviour of their solutions.

However, to what extent can we draw from the behaviour of the field equations under conformal rescalings of the metric conclusions on the large scale behaviour of the solution? The present article is trying to give some answer to this question. The latter might be rather aimless were it not for a concrete proposal pointing precisely into this direction. Motivated by earlier studies of the important physical question of how to characterize gravitational radiation without relying on approximations, R. Penrose suggested to analyses the asymptotic behaviour of gravitational fields in terms of extensions of the conformal structure to null infinity $(65,660$ ).

The subsequent attempt (23], 24]) to resolve the controversy concerning the assumptions made in [65] and to understand the smooth interplay between the conformal structure and the field equations suggested in [66] disclosed the very peculiar behaviour of Einstein's field equations under conformal rescalings. They turned out to be conformally regular and assume with this property a very special position between conformally singular equations like the massive KleinGordon equations on a given background and conformally invariant equations like the Maxwell or Yang-Mills equations in four dimensions. It will be the purpose of this article to explain the origin, the properties, and the consequences of the resulting conformal field equations.

Almost all the results described in the following have been obtained by considering as a guiding problem the question to what extent the assumptions underlying [65] can be justified. We are beginning to see now that this question proved fruitful because these assumptions refer to the key geometrical structure and draw such a sharp line between what is true and what isn't that they required, and still require, a most careful and detailed analysis. The results obtained so far indicate that in the most interesting case, that of a vanishing cosmological constant, the situation is much too subtle to simply allow us to say that the assumptions of [65] are right or wrong.

In the following we shall make a particular effort to sort out the delicate issues which arise here, hoping to put a controversy, whose 'arguments' too often relied more on personal taste than on insight, onto a rational basis. Such an effort is appropriate because due to the results which are available now the mathematical analysis is coming close to a point where the more subtle mathematical and physical questions concerning the foundations of the theory of isolated self gravitating systems become amenable to detailed discussions.

The investigations are now way beyond a situation which would allow us to arrive at reliable statements by investigations of the linearized equations, 'physical arguments' etc. In fact, it is difficult to develop any intuition on phys- 
ical grounds for the extreme questions about solutions of Einstein's equations which are being asked here. Consequently, most results are stated in the form of existence theorems (referred to in the following only in a non-technical way). The physicist mainly interested in wave forms and observational facts, who conceives of this as merely interesting mathematics, may be reminded that abstract statements about the existence of general classes of solutions with certain global properties usually only epitomize collections of results about details of the solutions, particular features of the equations, and new techniques with many more applications.

More importantly, it may be pointed out to him that the results alluded to above become of a practical use now, which was not anticipated in 65. As discussed in the articles by J. Frauendiener and S. Husa in this volume they form a basis for numerical calculations covering asymptotic parts of space-time. Most remarkably, though still to be explored in numerical work, they open the unique possibility to calculate numerically on finite grids maximal, globally hyperbolic, asymptotically flat solutions to Einstein's field equations, including their asymptotics in null directions and the radiation field at null infinity. These solutions will be determined solely and uniquely from standard Cauchy data (subject to certain, quite weak, conditions near space-like infinity) and require besides the idealization of asymptotic flatness no further approximation.

Working out the details of such truly global solutions, either analytical or with numerical methods, will still require a considerable amount of work and inventiveness. However, the main point to be made here is that both possibilities are open now and both approaches have a lot to gain from each other. Each of them will pose questions which will shed new light on problems of the other approach and the answers obtained in one of them will suggest new solutions to the other one.

A main open problem in this context is concerned with the question to what extent it will be possible to approach 'time-like infinity' within a numerical calculation if the solution develops singularities and event horizons. This problem has hardly been touched yet. Even for basic solutions like the Schwarzschild or the Kerr solution a careful and detailed analysis of time-like infinity in the conformal picture is not available yet. Some of these questions and also the related problem of gauge conditions will be addressed at the end of this article.

It is clear that there is still a long way to go before we will be able to obtain wave forms of sufficient generality or arrive at insights which will bring us closer to a resolution of the problem of cosmic censorship. Nevertheless, it appears that the interaction between numerical and analytical methods referred to above offers the best prospects for further progress.

Any work on general classes of solutions to the Einstein equations is necessarily of a very technical nature. In the following we shall give a survey on certain results and the current analytical work based on the conformal fields equations which emphasizes the general ideas. With the exception of the somewhat detailed discussion of the conformal field equations, technicalities will be kept at a minimum. 


\section{The conformal field equations}

The core of Einstein's theory of gravitation is represented by his field equations

$$
\tilde{R}_{\mu \nu}-\lambda \tilde{g}_{\mu \nu}=\kappa\left(T_{\mu \nu}-\frac{1}{2} T \tilde{g}_{\mu \nu}\right),
$$

for the metric $\tilde{g}_{\mu \nu}$, where $\lambda$ denotes the cosmological constant and $T_{\mu \nu}$ the energy momentum tensor. Most properties of the gravitational field should follow from these equations. Those which don't and have to be 'put in by hand' characterize general features as well as specific properties of the physical situation to be modeled. For instance, in the case of cosmological space-times one may assume compact time slices while in the description of isolated gravitating systems one will require the existence of Cauchy hypersurfaces with asymptotically flat ends, but in both cases one has the freedom to prescribe initial data in agreement with these assumptions. In the following we will mainly be interested in the second of these scenarios.

Equations (11) are known to be gauge hyperbolic in the sense that they are hyperbolic if suitable gauge conditions are imposed. Expressed in terms of coordinates $x^{\mu}$ satisfying the harmonicity condition $\tilde{\nabla}_{\nu} \tilde{\nabla}^{\nu} x^{\mu}=0$, equations (11) take the form

$$
\tilde{g}^{\alpha \beta} \partial_{\alpha} \partial_{\beta} \tilde{g}_{\mu \nu}=Q_{\mu \nu}(\tilde{g}, \partial \tilde{g}, T)
$$

of a system of wave equations for the metric coefficients $\tilde{g}_{\mu \nu}$, where the right hand side depends on the derivatives of these coefficients up to first order and on the energy momentum tensor.

The form (2) reveals the double role played by the light cone structure or, equivalently, the conformal structure. On the one hand the conformal structure is part of the unknown since it determines the metric up to a positive point dependent factor, on the other hand it defines the characteristic cone $\left\{\xi \in T^{*} M \mid \tilde{g}^{\alpha \beta} \xi_{\alpha} \xi_{\beta}=0\right\}$ of the form (2) of Einstein's equations (for this and various other general notions and results concerning the field equations we refer the reader to [45]). The characteristics strongly govern the local propagation properties of Einstein's equations. In fact, a more detailed argument involving (2) implies that equations (11) respect the local causality requirement of relativity, provided the equations for the matter fields entering the energy momentum tensor are chosen appropriately. This is related to the fact that the characteristic cone determines the domains of uniqueness for equation (17).

The tight relations between the structure of characteristics, the causal structure, and the light cone structure or, equivalently, the conformal structure, all of which depend on the solution at hand, is a very distinctive feature of Einstein's theory. However, the theory proposed by Einstein gives no reason to expect that the field equations would be particularly well behaved under conformal rescalings $\tilde{g}_{\mu \nu} \rightarrow g_{\mu \nu}=\Omega^{2} \tilde{g}_{\mu \nu}$. The equations are chosen to determine isometry classes of solutions and are thus not conformally covariant. Formally, this is 
seen from the transformation law of the Ricci tensor, given in $n$ dimensions by

$$
R_{\nu \rho}[g]=\tilde{R}_{\nu \rho}[\tilde{g}]-\frac{n-2}{\Omega} \nabla_{\nu} \nabla_{\rho} \Omega-g_{\nu \rho} g^{\lambda \delta}\left(\frac{1}{\Omega} \nabla_{\lambda} \nabla_{\delta} \Omega-\frac{n-1}{\Omega^{2}} \nabla_{\lambda} \Omega \nabla_{\delta} \Omega\right)
$$

where on the right hand side $\nabla$ denotes the connection associated with the metric $g$.

If Einstein's equation (11) is assumed in this relation and the conformal factor $\Omega$ is considered as a given function on the solution manifold, we obtain an equation for the conformal metric $g_{\nu \rho}$. It should be possible to analyse this equation by applying the techniques known for Einstein's equation, since the principal parts of the differential operators occurring in both equations, i.e. the parts containing the derivatives of highest order, are identical. For our intended application this equation for $g_{\nu \rho}$ has two deficiencies. The most conspicuous one is the occurrence of factors $1 / \Omega$, which makes the equation singular at the set $\mathcal{J}^{\prime}=\{\Omega=0\}$. Since this set will indicate some kind of infinity of the solution space-time, it will be of particular interest to us. The second problem arises because the function $\Omega$, in fact also the manifold underlying the solution and the set $\mathcal{J}^{\prime}$, are not given a priori. They are related to the global geometry of the solution and must be determined jointly with the metric.

Remarkably, Einstein's equations allow us to resolve these problems. They turn out to be conformally regular in the sense that there exist conformal representations of the equation which do not contain factors $\Omega^{-1}$ (resp. factors $\Omega$ in the principal part) and for which the conformal factor is determined by the equations themselves. To discuss this further we recall a few facts of conformal geometry.

\subsection{Conformal geometry}

A smooth conformal structure $\mathcal{C}$ on a manifold $M$ is given by a family of smooth locally defined metrics whose domains of definition exhaust $M$ and which are related on the intersections of these domains by conformal rescalings with positive conformal factors. A smooth pseudo-Riemannian space $(M, g)$ uniquely determines a smooth conformal structure on $M$ which we denote by $\mathcal{C}_{g}$. Using a suitable partition of unity, it can be shown that any conformal structure can be obtained this way and we shall often represent a conformal structure in terms of a globally defined metric in the conformal class.

A Lorentzian conformal structure $\mathcal{C}$ determines a null cone at each point of $M$. Conversely, the null cones fix $\mathcal{C}$ uniquely (cf. [52]). As a consequence 'spaceand time-like' are well defined for $\mathcal{C}$ and a few further important space-time structures are implied. A null hypersurface for $\mathcal{C}$ is a smooth (say) hypersurface which at each of its points is tangent to the null cone field. The unique directions of tangency can be integrated to obtain null curves which rule the null hypersurface. Considered as point sets, these curves coincide with null geodesics of the metrics in the conformal class and any null geodesic can locally be obtained this way. Extending the null geodesics generating a null hypersurface in any of 
their affine parameterizations we obtain an unique extension. The resulting set, called a wave front, will in general not any longer be a smooth hypersurface, because families of null geodesics tend to develop caustics, i.e. form envelopes or intersections. The wave fronts and their caustics are also invariants of the conformal structure.

On the level of local differential geometry the most important invariant of a conformal structure $\mathcal{C}_{g}$ is its conformal Weyl tensor $C^{\mu}{ }_{\nu \lambda \rho}$ (observe the index positions), which is the trace-free part in the decomposition

$$
R_{\nu \lambda \rho}^{\mu}=2\left\{g^{\mu}{ }_{[\lambda} L_{\rho] \nu}-g_{\nu[\lambda} L_{\rho]}^{\mu}\right\}+C_{\nu \lambda \rho}^{\mu},
$$

of the curvature tensor of $g$, where the trace parts are represented by the tensor

$$
L_{\mu \nu}=\frac{1}{n-2}\left(R_{\mu \nu}-\frac{1}{2(n-1)} R g_{\mu \nu}\right) \text {. }
$$

In terms of the Levi-Civita connection $\nabla$ of $g$ and some coordinate basis $\partial_{\mu}$ our conventions are such that $R^{\mu}{ }_{\nu \lambda \rho} X^{\nu}=\left(\nabla_{\lambda} \nabla_{\rho}-\nabla_{\rho} \nabla_{\lambda}\right) X^{\mu}$. We denote by $R_{\mu \nu}=R_{\mu \rho \nu}^{\rho}$ and $R$ the Ricci tensor and the Ricci scalar of $g$ respectively.

The class of torsion free covariant derivative operators naturally associated with a conformal class $\mathcal{C}$ comprises but is larger than the set of Levi-Civita operators for the metrics representing $\mathcal{C}$. A Weyl connection for $\mathcal{C}_{g}$ is a torsion free connection $\hat{\nabla}$ on $M$ which respects the conformal class $\mathcal{C}_{g}$ in the following sense. For any $C^{1}$ curve $x(\lambda)$ defined on a neighbourhood of the origin of $\mathbb{R}$ and any frame field $e_{k}, k=1, \ldots, n$, which is parallelly transported along $x(\lambda)$ with respect to $\hat{\nabla}$, there exists a function $\Omega_{\lambda}>0$ along $x(\lambda)$ such that $\left.g\left(e_{i}, e_{k}\right)\right|_{x(\lambda)}=\left.\Omega_{\lambda}^{2} g\left(e_{i}, e_{k}\right)\right|_{x(0)}$. It follows from this that

$$
\hat{\nabla}_{\rho} g_{\mu \nu}=-2 f_{\rho} g_{\mu \nu}
$$

with some arbitrary 1-form $f$ on $M$ and that the connection coefficients defined by $\nabla_{\partial_{\mu}} \partial_{\nu}=\Gamma_{\mu}{ }^{\rho}{ }_{\nu} \partial_{\rho}$ and $\hat{\nabla}_{\partial_{\mu}} \partial_{\nu}=\hat{\Gamma}_{\mu}{ }^{\rho}{ }_{\nu} \partial_{\rho}$ are related by

$$
\hat{\Gamma}_{\mu}{ }^{\rho}{ }_{\nu}=\Gamma_{\mu}{ }^{\rho}{ }_{\nu}+S(f)_{\mu}{ }^{\rho}{ }_{\nu}, \quad \text { with } \quad S(f)_{\mu}{ }^{\rho}{ }_{\nu} \equiv \delta^{\rho}{ }_{\mu} f_{\nu}+\delta^{\rho}{ }_{\nu} f_{\mu}-g_{\mu \nu} g^{\rho \lambda} f_{\lambda} \text {. }
$$

In the special case where the 1 -form $f$ is exact such that it can be written on suitable open sets $U$ in the form

$$
f=-\Omega^{-1} d \Omega
$$

with some smooth function $\Omega>0$, the connection $\hat{\nabla}$ coincides on $U$ with the Levi-Civita connection $\tilde{\nabla}$ of the metric $\tilde{g}$ satisfying $g_{\mu \nu}=\Omega^{2} \tilde{g}_{\mu \nu}$. by

The curvature tensors of the connections $\hat{\nabla}$ and $\nabla$ satisfying (阬) are related

$$
\begin{aligned}
& \hat{R}_{\nu \lambda \rho}^{\mu}-R_{\nu \lambda \rho}^{\mu}=2\left\{\nabla_{[\lambda} S_{\rho]}{ }_{\nu}^{\mu}+S_{\delta}{ }^{\mu}{ }_{[\lambda} S_{\rho]}{ }^{\delta}{ }_{\nu}\right\}=2\left\{g^{\mu}{ }_{[\rho} \nabla_{\lambda]} f_{\nu}\right. \\
& \left.\quad+\nabla_{[\rho} f^{\mu} g_{\lambda] \nu}-g^{\mu}{ }_{\nu} \nabla_{[\rho} f_{\lambda]}-g^{\mu}{ }_{[\rho} f_{\lambda]} f_{\nu}+g_{\nu[\rho} f_{\lambda]} f^{\mu}+g^{\mu}{ }_{[\rho} g_{\lambda] \nu} f_{\delta} f^{\delta}\right\},
\end{aligned}
$$


were indices are raised or lowered with respect to $g$. This implies for the Ricci tensor $\hat{R}_{\nu \rho}=\hat{R}_{\nu \mu \rho}^{\mu}$ and the Ricci scalar $\hat{R}=g^{\nu \rho} \hat{R}_{\nu \rho}$

$$
\begin{gathered}
\hat{R}_{\nu \rho}=R_{\nu \rho}-(n-1) \nabla_{\rho} f_{\nu}+\nabla_{\nu} f_{\rho}+(n-2) f_{\nu} f_{\rho}-g_{\nu \rho}\left(\nabla_{\lambda} f^{\lambda}+(n-2) f_{\lambda} f^{\lambda}\right), \\
\hat{R}=R-2(n-1) \nabla_{\lambda} f^{\lambda}-(n-1)(n-2) f_{\lambda} f^{\lambda} .
\end{gathered}
$$

Setting here $f=-\Omega^{-1} d \Omega$, we obtain for the metrics $g, \tilde{g}$ which are related by the rescaling $g=\Omega^{2} \tilde{g}$ the relation (3) of their Ricci tensors, which implies for their Ricci scalars the relation

$$
4 \frac{n-1}{n-2} \nabla_{\mu} \nabla^{\mu} \theta-R[g] \theta=-\tilde{R}[\tilde{g}] \theta^{\frac{n+2}{n-2}} .
$$

For later convenience we set here $\theta=\Omega^{-\frac{n-2}{2}}$.

The analogue of the decomposition (㺼 takes the form

$$
\hat{R}^{\mu}{ }_{\nu \lambda \rho}=2\left\{g^{\mu}{ }_{[\lambda} \hat{L}_{\rho] \nu}-g^{\mu}{ }_{\nu} \hat{L}_{[\lambda \rho]}-g_{\nu[\lambda} \hat{L}_{\rho]}{ }^{\mu}\right\}+C^{\mu}{ }_{\nu \lambda \rho},
$$

where the trace parts of the curvature tensor are represented by the tensor

$$
\hat{L}_{\mu \nu}=\frac{1}{n-2}\left(\hat{R}_{(\mu \nu)}-\frac{n-2}{n} \hat{R}_{[\mu \nu]}-\frac{1}{2(n-1)} g_{\mu \nu} \hat{R}\right) .
$$

It is related to (5) by

$$
\nabla_{\mu} f_{\nu}-f_{\mu} f_{\nu}+\frac{1}{2} g_{\mu \nu} f_{\lambda} f^{\lambda}=L_{\mu \nu}-\hat{L}_{\mu \nu}
$$

By a straightforward calculation it can be verified that (5), (11) satisfy the identity

$$
\hat{\nabla}_{\rho} \hat{L}_{\mu \nu}-\hat{\nabla}_{\mu} \hat{L}_{\rho \nu}=\nabla_{\rho} L_{\mu \nu}-\nabla_{\mu} L_{\rho \nu}+f_{\lambda} C^{\lambda}{ }_{\nu \rho \mu} .
$$

\subsubsection{Conformal geodesics}

We have seen that null geodesics, considered as point sets, are invariants of a Lorentzian conformal structure. With any conformal structure $\mathcal{C}_{\tilde{g}}$ is associated in fact a much larger class of distinguished curves called conformal geodesics. We shall only give an outline of their properties here, referring the reader to [38, [41], and [46] for details and further results. A conformal geodesic curve $x(\tau)$ is obtained, together with a 1-form $b=b(\tau)$ along the curve, as a solution to the system of equations

$$
\begin{gathered}
\left(\tilde{\nabla}_{\dot{x}} \dot{x}\right)^{\mu}+S(b)_{\lambda}{ }_{\rho}{ }_{\rho} \dot{x}^{\lambda} \dot{x}^{\rho}=0 \\
\left(\tilde{\nabla}_{\dot{x}} b\right)_{\nu}-\frac{1}{2} b_{\mu} S(b)_{\lambda}{ }_{\nu}{ }_{\nu} \dot{x}^{\lambda}=\tilde{L}_{\lambda \nu} \dot{x}^{\lambda},
\end{gathered}
$$

where $\tilde{L}$ and $S$ are as in (5), (7). There are more conformal geodesics than geodesics for any metric in the conformal class because for given initial data 
$x_{*} \in M, \dot{x}_{*} \in T_{x_{*}} M, b_{*} \in T_{x_{*}}^{*} M$ there exists a unique conformal geodesic $x(\tau)$, $b(\tau)$ near $x_{*}$ satisfying for given $\tau_{*} \in \mathbb{R}$

$$
x\left(\tau_{*}\right)=x_{*}, \quad \dot{x}\left(\tau_{*}\right)=\dot{x}_{*}, \quad b\left(\tau_{*}\right)=b_{*} .
$$

The sign of $\tilde{g}(\dot{x}, \dot{x})$ is preserved along a conformal geodesic but not its modulus. Conformal geodesics, considered as point sets, are in general different from the metric geodesics of a metric in the conformal class. Furthermore, they admit general fractional linear maps as parameter transformations. Initial data with $\tilde{g}\left(\dot{x}_{*}, \dot{x}_{*}\right)=0, \dot{x}_{*} \neq 0$, determine null curves which coincide, as point sets, with the null geodesics of the metrics in the conformal class. The fact that we shall mainly make use of time-like conformal geodesics in the following, should not be taken as an indications that null or space-like conformal geodesics can not be useful.

Conformal geodesics are conformal invariants in the following sense. Denote by $f$ a smooth 1 -form field. Then, if $x(\tau), b(\tau)$ solve the conformal geodesics equations (14), (15), the pair $x(\tau), b(\tau)-\left.f\right|_{x(\tau)}$ solves the same equations with $\tilde{\nabla}$ replaced by the connection $\hat{\nabla}=\tilde{\nabla}+S(f)$ and $L$ by $\hat{L}$, i.e. $x(\tau)$, in particular its parameter $\tau$, is independent of the Weyl connection in the conformal class which is used to write the equations.

Assume that there is given a smooth congruence of conformal geodesics covering an open set $U$ of our manifold such that the associated 1-forms $b$ define a smooth field on $U$. Denote by $\hat{\nabla}$ the torsion free connection on $U$ which defines with the connection entering (14), (15) the difference tensor $\hat{\nabla}-\tilde{\nabla}=S(b)$ and denote by $\hat{L}$ the tensor (11) derived from $\hat{\nabla}$. Comparing with (12), we find that equations (14), (15) can be written

$$
\begin{gathered}
\hat{\nabla}_{\dot{x}} \dot{x}=0, \\
\hat{L}_{\lambda \nu} \dot{x}^{\lambda}=0 .
\end{gathered}
$$

Let $e_{k}$ be a frame field satisfying

$$
\hat{\nabla}_{\dot{x}} e_{k}=0
$$

along the congruence, and suppose that $S$ is a hypersurface transverse to the congruence which meets each of the curves exactly once and on which $\tilde{g}\left(e_{i}, e_{k}\right)=$ $\Theta_{*}^{2} \eta_{i k}$ with some function $\Theta_{*}>0$. Following the argument which leads to (6) we see that $\tilde{g}\left(e_{i}, e_{k}\right)=\Theta^{2} \eta_{i k}$ on $U$ with a function $\Theta$ satisfying

$$
\hat{\nabla}_{\dot{x}} \Theta=\Theta<\dot{x}, b>,\left.\quad \Theta\right|_{S}=\Theta_{*} .
$$

\subsection{Derivation of the conformal field equations}

The curvature tensor always satisfies the Bianchi identity $\nabla_{[\delta} R^{\mu}{ }_{|\nu| \lambda \rho]}=0$. Observing (㺼, we can write it in $n \geq 3$ dimensions in the form

$$
\nabla_{[\delta} C^{\mu}{ }_{|\nu| \lambda \rho]}=2\left\{g_{\nu[\lambda} \nabla_{\delta} L_{\rho]}^{\mu}-g^{\mu}{ }_{[\lambda} \nabla_{\delta} L_{\rho] \nu}\right\},
$$


which gives after a contraction

$$
\nabla_{\mu} C^{\mu}{ }_{\nu \lambda \rho}=(n-3)\left\{\nabla_{\lambda} L_{\rho \nu}-\nabla_{\rho} L_{\lambda \nu}\right\} .
$$

We assume now that the physical metric $\tilde{g}$ satisfies Einstein's field equation (11) and try to express these equations in terms of the conformal metric

$$
g=\Omega^{2} \tilde{g},
$$

and the conformal factor $\Omega>0$ such that the difficulties pointed out above are avoided. Although our discussion can be generalized to include certain matter fields (cf. for instance [32], [53] and also, for an analysis with a different intention and different results, P. Tod, this volume), we assume for simplicity that matter fields are absent, i.e. $T_{\mu \nu}=0$. Equation (22) reduces then to

$$
\tilde{\nabla}_{\mu} C^{\mu}{ }_{\nu \lambda \rho}=0 .
$$

This equation is of particular interest for us because due to the identity

$$
\nabla_{\mu}\left(\Omega^{3-n} C^{\mu}{ }_{\nu \lambda \rho}\right)=\Omega^{3-n} \tilde{\nabla}_{\mu} C^{\mu}{ }_{\nu \lambda \rho},
$$

which holds for any conformal factor, it has a certain conformal covariance. It implies for

$$
d^{\mu}{ }_{\nu \lambda \rho}=\Omega^{3-n} C^{\mu}{ }_{\nu \lambda \rho},
$$

the Bianchi equation

$$
\nabla_{\mu} d_{\nu \lambda \rho}^{\mu}=0
$$

which, in a sense, represents the core of all the systems to be considered in the following. We consider now equation (4) for the conformal metric $g$ and write it in the form

$$
R^{\mu}{ }_{\nu \lambda \rho}=2\left\{g^{\mu}{ }_{[\lambda} L_{\rho] \nu}-g_{\nu[\lambda} L_{\rho]}^{\mu}\right\}+\Omega^{n-3} d^{\mu}{ }_{\nu \lambda \rho} .
$$

Using (22) also for $g$ and observing (25), (26), we obtain for $L_{\mu \nu}$, which represents the Ricci tensor of the conformal metric, the equation

$$
\nabla_{\lambda} L_{\rho \nu}-\nabla_{\rho} L_{\lambda \nu}=\Omega^{n-4} \nabla_{\mu} \Omega d^{\mu}{ }_{\nu \lambda \rho} .
$$

For later discussion we note how (27), (28) generalize if we admit besides rescalings also transitions to Weyl connections which are related to the LeviCivita connection $\nabla$ of $g$ by difference tensors

$$
\hat{\nabla}-\nabla=S(f),
$$

with smooth 1-forms $f$. Since $\nabla-\tilde{\nabla}=S\left(\Omega^{-1} \nabla \Omega\right)$, it follows that $\hat{\nabla}-\tilde{\nabla}=$ $S\left(\Omega^{-1} d\right)$ with the smooth 1-form $d_{\mu}=\Omega f_{\mu}+\nabla_{\mu} \Omega$. By (10) equation (27) is then replaced by

$$
\hat{R}_{\nu \lambda \rho}^{\mu}=2\left\{g^{\mu}{ }_{[\lambda} \hat{L}_{\rho] \nu}-g^{\mu}{ }_{\nu} \hat{L}_{[\lambda \rho]}-g_{\nu[\lambda} \hat{L}_{\rho]}^{\mu}\right\}+\Omega^{n-3} d^{\mu}{ }_{\nu \lambda \rho},
$$


while (28) and (13) imply the equation

$$
\hat{\nabla}_{\rho} \hat{L}_{\mu \nu}-\hat{\nabla}_{\mu} \hat{L}_{\rho \nu}=\Omega^{n-4} d_{\mu} d_{\nu \lambda \rho}^{\mu} .
$$

By admitting rescalings and transitions to Weyl connections we have artificially introduced a conformal gauge freedom into the equations. If these equations are to be used to derive information about the physical metric $\tilde{g}$ by analyzing boundary value problems, this freedom has to be removed again by imposing gauge conditions such that (i) the resulting equations ensure that $\tilde{g}$, derived by (23) from $\Omega$ and $g$, satisfies the field equations, (ii) interesting information on $\tilde{g}$ can be gained by suitably fixing $\Omega$ and $f$. Two quite different methods have been discussed so far (23], 24, [35]).

\subsubsection{The metric conformal field equations}

We consider only conformal rescalings of the metric and try to get equations which allow us to specify the conformal factor in a useful way. If we observe (11) in the form $\tilde{R}_{\mu \nu}=\frac{1}{n} \tilde{R} \tilde{g}_{\mu \nu}$ in (3), the resulting equation, which we read now as equation for $\Omega$, can be written in the form

$$
\nabla_{\mu} \nabla_{\nu} \Omega=-\Omega L_{\mu \nu}+s g_{\mu \nu}
$$

with

$$
s=\frac{1}{n} \nabla_{\lambda} \nabla^{\lambda} \Omega+\frac{1}{2 n(n-1)} R \Omega .
$$

Equation (32) is obviously overdetermined. If we apply $\nabla_{\rho}$ to both sides, commute $\nabla_{\rho}$ with $\nabla_{\mu}$, contract over $\rho$ and $\nu$, and use the twice contracted Bianchi identity, we obtain the integrability condition

$$
\nabla_{\mu} s=-\nabla^{\nu} \Omega L_{\nu \mu}
$$

which we read as an equation for $s$.

To see the role of the cosmological constant, we observe that the transformation law of the Ricci scalar gives

$$
\lambda=(n-1)\left(2 \Omega s-\nabla_{\rho} \Omega \nabla^{\rho} \Omega\right) .
$$

Equations (32), (33) then imply for the quantity on the right hand side

$$
\begin{gathered}
\frac{1}{2} \nabla_{\lambda}\left(2 \Omega s-\nabla_{\rho} \Omega \nabla^{\rho} \Omega\right)=\nabla_{\lambda} \Omega s+\Omega \nabla_{\lambda} s-\nabla^{\rho} \Omega \nabla_{\rho} \nabla_{\lambda} \Omega \\
=\nabla_{\lambda} \Omega s+\Omega\left(-\nabla^{\nu} \Omega L_{\nu \lambda}\right)-\nabla^{\rho} \Omega\left(-\Omega L_{\rho \lambda}+g_{\rho \lambda} s\right)=0,
\end{gathered}
$$

which reflects the well known fact that the twice contracted Bianchi identity implies that $\lambda$ must be constant. It follows that (34) will be a consequence of the other equations if it is arranged to hold at one point by a suitable choice of the initial data. 
We consider (26), (27), (28), (32), (33), (34) as equations for the unknown tensor fields

$$
g_{\mu \nu}, \Omega, s, L_{\mu \nu}, d^{\mu}{ }_{\nu \lambda \rho},
$$

and refer to them as the metric conformal field equations. These equations hold for any vacuum solution $\tilde{g}$ and any conformal factor $\Omega$.

A way to impose a gauge condition on the conformal factor is suggested by the following observation. If $g$ is any metric and $R^{*}$ a given function, the metric $g^{*}=\theta^{\frac{4}{n-2}} g$ will have by (9) the Ricci scalar $R\left[g^{*}\right]=R^{*}$, if $\theta$ solves the equation

$$
4 \frac{n-1}{n-2} \nabla_{\mu} \nabla^{\mu} \theta-R[g] \theta=-R^{*} \theta^{\frac{n+2}{n-2}} .
$$

Since locally this equation can always be solved for a positive function $\theta$, it follows that locally the Ricci scalar $R$ of the conformal metric can be prescribed in the metric conformal field equations as a gauge source function for the conformal scaling. Its choice will depend on the given situation and the choices of the other gauge dependent quantities. We can set $R=0$ but, depending on the circumstances, there may be better choices. In any case this leaves the freedom to specify suitable boundary data for the conformal factor.

From the way we obtained the metric conformal field equations it is clear that they are equivalent to the vacuum field equations (ignoring questions of smoothness) where $\Omega$ is positive but that they make sense also where $\Omega$ vanishes. While the system is regular in this sense, it is also complicated and highly overdetermined. We shall see below that the formal regularity of the equations does not necessarily imply that the data must be regular nor does it ensure by itself that the asymptotic behaviour of the solutions can be controlled conveniently. To get an insight into this one needs to work out suitable initial value problems in great detail and control the constraints as well as the evolution. We now take a look at the various parts of the system.

\subsubsection{The Bianchi equation}

For a solution $\tilde{g}$ to the vacuum field equations the Bianchi identity (21) takes the form

$$
\tilde{\nabla}_{\delta} C^{\mu}{ }_{\nu \lambda \rho}+\tilde{\nabla}_{\rho} C^{\mu}{ }_{\nu \delta \lambda}+\tilde{\nabla}_{\lambda} C^{\mu}{ }_{\nu \rho \delta}=0 .
$$

Contracting with $\tilde{\nabla}_{\delta}$, commuting derivatives, and observing (24), we obtain

$\tilde{\nabla}_{\delta} \tilde{\nabla}^{\delta} C^{\mu}{ }_{\nu \tau \rho}=2\left\{C^{\mu}{ }_{\pi \delta \rho} C^{\pi}{ }_{\nu \tau}{ }^{\delta}+C^{\mu}{ }_{\pi \tau \delta} C^{\pi}{ }_{\nu \rho}{ }^{\delta}+C^{\mu}{ }_{\nu \pi \delta} C^{\pi}{ }_{\tau \rho}{ }^{\delta}\right\}+2 \lambda C^{\mu}{ }_{\nu \tau \rho}$.

Thus, in the Lorentzian case, which will be considered in the following, we find the well known fact that the Bianchi identity implies a system of hyperbolic equations for the conformal Weyl tensor in any dimension $n \geq 4$.

In $n=4$ dimensions we can define the left and right dual of the conformal Weyl tensor, given by ${ }^{*} C_{\mu \nu \tau \rho}=\frac{1}{2} \tilde{\epsilon}_{\mu \nu}{ }^{\delta \pi} C_{\delta \pi \tau \rho}$ and $C_{\mu \nu \tau \rho}^{*}=\frac{1}{2} \tilde{\epsilon}_{\tau \rho}{ }^{\delta \pi} C_{\mu \nu \delta \pi}$ respectively, and it is well known that they coincide. It follows that

$$
\tilde{\epsilon}_{\pi}{ }^{\delta \tau \rho} \tilde{\nabla}_{\delta} C^{\mu}{ }_{\nu \tau \rho}=2 \tilde{\nabla}_{\delta} C^{* \mu}{ }_{\nu \pi}{ }^{\delta}=2 \tilde{\nabla}_{\delta}{ }^{*} C^{\mu}{ }_{\nu \pi}{ }^{\delta}=-\tilde{\epsilon}^{\mu}{ }_{\nu}{ }^{\tau \rho} \tilde{\nabla}_{\delta} C^{\delta}{ }_{\pi \tau \rho},
$$


which implies that the contracted Bianchi identity (24) is equivalent to the Bianchi identity (35). It follows that (24) and, by the same argument equation (26), imply hyperbolic equations.

In dimensions $n \geq 5$ the situation is quite different as can be seen by a simple function counting. The conformal Weyl tensor has in $n$ dimensions

$$
\frac{1}{12} n^{2}\left(n^{2}-1\right)-\frac{1}{2} n(n+1),
$$

independent components while the contracted Bianchi identity (24) provides

$$
\frac{1}{3} n(n-2)(n+2),
$$

independent equations. Moreover, if we express (24) in Gauss coordinates with $x^{0}$ the affine parameter on the geodesics orthogonal to the space-like hypersurfaces $\left\{x^{0}=\right.$ const. $\}$, it follows that the equations $\tilde{\nabla}_{\delta} C^{\delta}{ }_{0 \lambda \rho}=0$ involve only derivatives in the directions $\partial_{x^{1}}, \ldots, \partial_{x^{n-1}}$ and thus provide

$$
\frac{1}{2} n(n-1)
$$

constraints. Consequently, there are not enough equations in (24) if $n \geq 6$ while for $n=5$ there are as many equations as unknowns but not sufficiently many evolution equations. It follows that a system of conformal equations which includes as equation for the rescaled conformal Weyl tensor only the Bianchi equation provides a good evolution system only in four dimensions.

If we use in $n \geq 5$ dimensions the full Bianchi identities to derive a system of wave equations for $d^{\mu}{ }_{\nu \lambda \rho}$, it appears that not all terms of the form $\Omega^{-1} \nabla_{\mu} \Omega$ can be removed from the equations. It would be interesting to know whether there exist other conformally covariant equations which could replace the Bianchi equation in higher dimensions. If it turns out that a conformally regular representation for the Einstein equation can only be obtained in four dimensions, it would also be interesting to know whether there is a principal difference in the asymptotic behaviour of the fields in four and higher dimensional space-times.

We shall restrict our discussion in the following to the case of four dimensions.

Instead of expressing the intrinsic hyperbolicity of the Bianchi equation in terms of a system of wave equations we work directly with the first order system. The benefits of this will be seen later. There are known two quite different possibilities to make the hyperbolicity of the equations manifest, both of them have been used in numerical calculations (cf. J. Frauendiener and S. Husa, this volume). It has been shown in [37] how to obtain hyperbolic equations from the Bianchi equation in the ADM representation of the metric. The other method, which is based on a frame formalism, has many advantages and can be adapted to various geometrical situations. We can use real orthonormal frames (cf. 44]) but most convenient are pseudo-orthonormal frames or spin frames. In the spin 
frame formalism the equations split almost automatically into constraints and (symmetric hyperbolic) evolution equations (cf. [23], 227, [32]). We shall use this fact here to discuss certain features of the Bianchi equation.

In the spin frame formalism we have a frame field $e_{a a^{\prime}}, a, a^{\prime}=0,1$, with $e_{00^{\prime}}, e_{11^{\prime}}$ real null vector fields and $e_{01^{\prime}}, e_{10^{\prime}}$ complex vector fields which are conjugate to each other. Their only non-vanishing scalar products are given by $g\left(e_{00^{\prime}}, e_{11^{\prime}}\right)=1, g\left(e_{01^{\prime}}, e_{10^{\prime}}\right)=-1$. In the following discussion it is useful to think of these vector fields as being obtained in the form

$$
\begin{gathered}
e_{00^{\prime}}=\frac{1}{\sqrt{2}}\left(e_{0}+e_{3}\right), \quad e_{11^{\prime}}=\frac{1}{\sqrt{2}}\left(e_{0}-e_{3}\right), \\
e_{01^{\prime}}=\frac{1}{\sqrt{2}}\left(e_{1}+i e_{2}\right), \quad e_{10^{\prime}}=\frac{1}{\sqrt{2}}\left(e_{1}-i e_{2}\right),
\end{gathered}
$$

from a frame field $e_{k}, k=0, \ldots, 3$, satisfying $g\left(e_{i}, e_{k}\right)=\eta_{i k}$.

The rescaled conformal Weyl tensor is represented by a completely symmetric spinor field $\phi_{a b c d}$, and the Bianchi equation by the equation

$$
0=\Lambda_{a b c a^{\prime}} \equiv \nabla^{f}{ }_{a^{\prime}} \phi_{a b c f},
$$

where $\nabla_{a a^{\prime}}$ denotes the covariant derivative on spinor fields in the direction of $e_{a a^{\prime}}$ and the index is raised with the antisymmetric spinor field $\epsilon^{a b}$ which satisfies $\epsilon^{01}=1$.

If we set $\Lambda_{a b c d}=\Lambda_{a b c a^{\prime}} \tau_{d} a^{\prime}$ with $\tau_{a}{ }^{a^{\prime}}=\delta_{a}{ }^{1} \delta_{0^{\prime}} a^{\prime}-\delta_{a}{ }^{0} \delta_{1^{\prime}}{ }^{a^{\prime}}$ we get the decomposition $\Lambda_{a b c d}=\Lambda_{(a b c d)}-\frac{3}{4} \epsilon_{d(c} \Lambda_{a b) f}{ }^{f}$ into its irreducible parts

$$
-2 \Lambda_{(a b c d)}=P \phi_{a b c d}-2 \mathcal{D}_{(d}^{f} \phi_{a b c) f}, \quad \Lambda_{a b f}^{f}=\mathcal{D}^{e f} \phi_{a b e f} .
$$

Here $P=\tau^{a a^{\prime}} \nabla_{a a^{\prime}}=\sqrt{2} \nabla_{e_{0}}$ and $\mathcal{D}_{a b}=\tau_{(a}{ }^{a^{\prime}} \nabla_{b) a^{\prime}}$ denote covariant directional derivatives such that $\mathcal{D}_{00}=-\nabla_{01^{\prime}}, \mathcal{D}_{01}=\mathcal{D}_{10}=\frac{1}{\sqrt{2}} \nabla_{e_{3}}, \mathcal{D}_{11}=\nabla_{10^{\prime}}$ (cf. 32], [35] for more details of the underlying space-spinor formalism).

In a Cauchy problem one will in general assume $e_{0}$ to be the future directed normal to the initial hypersurface $S$. The operators $\mathcal{D}_{a b}$ then involve only differentiation in directions tangent to $S$ and the equations $\Lambda_{a b f}{ }^{f}=0$ are interior equations on $S$. They represent the six real constraint equations implied on $S$ by the Bianchi equation.

The remaining equations for $\phi_{a b c d}$ should be evolution equations. Multiplying the equations by suitable binomial coefficients (and considering the frame field and the connection coefficients as given), we find that the system

$$
-\left(\begin{array}{c}
4 \\
a+b+c+d
\end{array}\right) \Lambda_{(a b c d)}=0
$$

is in fact symmetric hyperbolic, i.e. it has the form

$$
A^{\mu} \partial_{\mu} u=H(x, u)
$$


with a $\mathbb{C}^{N}$-valued unknown $u$, a $\mathbb{C}^{N}$-valued function $H(x, u)$, and $N \times N$-matrixvalued, possibly $u$-dependent functions $A^{\mu}$ which are hermitian, i.e. ${ }^{T} \bar{A}^{\mu}=A^{\mu}$, and for which there exists at each point $x$ a covector $\xi_{\mu}$ such that $A^{\mu} \xi_{\mu}$ is positive definite. There exists a well developed theory for such systems (cf. [48], [58], [77, and also H.-O. Kreiss and O. Ortiz, this volume).

While the constraints implied on a given space-like hypersurface are determined uniquely, there is a large freedom to select useful evolution systems. In the present case it turns out that any system of the form

$$
\begin{gathered}
0=2 a \Lambda_{\left(0001^{\prime}\right)}, \\
0=(c-d) \Lambda_{\left(0011^{\prime}\right)}-2 a \Lambda_{\left(0000^{\prime}\right)}, \\
0=(c+d) \Lambda_{\left(0111^{\prime}\right)}-(c-d) \Lambda_{\left(0010^{\prime}\right)}, \\
0=2 e \Lambda_{\left(1111^{\prime}\right)}-(c+d) \Lambda_{\left(0110^{\prime}\right)}, \\
0=-2 e \Lambda_{\left(1110^{\prime}\right)},
\end{gathered}
$$

with $a, c, e>0$ and $-(2 e+c)<d<2 a+c$, is symmetric hyperbolic (the system (37) occurs here as a special case). This flexibility allows the Bianchi equation to be adapted to various geometrical situations such as characteristic initial value problems (23], [24], [25], [28], [57]), standard Cauchy problems (30], [29], [32]), and initial boundary value problems ([35], [44]). Note that for these systems the set of characteristics, given by the hypersurfaces of the form $\{f=0, d f \neq 0\}$ with $f \in C^{1}(M)$ on which $\operatorname{det}\left(A^{\mu} f_{, \mu}\right)=0$ is satisfied at each of their points, does not only comprise the null hypersurfaces and can in fact be quite complicated.

\subsubsection{The constraints}

Related to the gauge freedom is the fact that, written in suitably adapted coordinates (and frame field, if used), Einstein's equations as well as the metric conformal field equations imply inner equations, i.e. constraints, on any time-like, null, or space-like hypersurface. We will consider in the following the latter two cases, because they are particularly important in initial value problems. (Timelike hypersurfaces are of interest in initial-boundary value problems, however, even in general analytical discussions of this problem a detailed discussion of the constraints on these hypersurfaces is not necessarily required [44].)

Null hypersurfaces representing the physical characteristics of the Einstein equations, the system of inner equations is quite different in nature from those arising in the non-null case. There are on the one hand the intrinsic equations arising because of the gauge freedom and on the other hand the characteristic inner equations arising due to the intrinsic wave equation character of the field equations and these equations combine in a very peculiar way. Let us consider a situation were data are to be prescribed on a null hypersurface $N$ and another hypersurface which has a space-like intersection $\Sigma$ with $N$. Then some of the unknowns can be prescribed completely freely on $N$. All the remaining unknowns can then be calculated on $N$ in suitably adapted coordinates from certain data 
on $\Sigma$ by solving a certain hierarchy of inner equations on $N$, each member of which reduces to a system of ODEs along the null generators at their subsequent integration. Those equations which are not used in this procedure will then be satisfied on $N$ if they hold on $\Sigma$ (cf. [75] for the vacuum case).

The enormous simplification of the equations resulting from these facts in a suitably adapted gauge and the possibility to integrate the equations along characteristics 'out to infinity' led a number of groups to develop very efficient characteristic numerical computer codes (cf. R. Bartnik and A. Norton, J. Font, L. Lehner, this volume).

Though being more complicated, the inner equations induced on null hypersurfaces by the metric conformal field equations have a similar structure and, besides the data required to fix the conformal gauge, the free data are, of course, up to a rescaling the same $(24)$.

In the following we will mainly be concerned with Cauchy problems and therefore consider now space-like initial hypersurfaces. The standard constraints induced on space-like hypersurfaces by the vacuum field equations form a system of underdetermined elliptic quasi-linear equations for the data induced by the 4 -metric on the initial hypersurface, i.e. the inner metric $\tilde{h}_{\alpha \beta}$ and the extrinsic curvature $\tilde{\chi}_{\alpha \beta}$. There exists a detailed theory for these equations which, under the assumption of constant mean extrinsic curvature, reduces the problem of solving the constraints to a problem of solving a linear elliptic system and a semi-linear elliptic scalar equation (cf. [12], [13]).

The metric conformal field equations imply a system of conformal constraints (26], [35]) on a space-like hypersurface $S$ which comprises the usual vacuum constraints but which is more complicated. This is not only due to the artificial scaling freedom which has been introduced into the vacuum equations but also because the equations include integrability conditions and the Gauss-Codazzi equations. There appear to be two ways of dealing with these equations. The first method solves essentially the standard vacuum constraints and calculates from the vacuum data the conformal data after introducing a suitable conformal rescaling. This method, which has been analyzed with a certain completeness and underlies the present numerical calculations, introduces certain numerical difficulties (cf. J. Frauendiener and S. Husa, this volume). A second possibility would be to solve the conformal constraints directly. First steps in a systematic investigation of this approach are reported in the article by A. Butscher in this volume (cf. also the discussion in [33]).

Any of these approaches has to deal with a problem which is not considered in the usual discussions of the standard vacuum constraints. In the situations which are most interesting for our applications the initial hypersurface contains a subset $\Sigma$, not belonging to the physical space-time, where the conformal factor vanishes. As seen in detail in the next sections, this set can be a 2-dimensional boundary which represents points at null infinity (cf. L. Andersson, this volume) or consist of a number of discrete compactification points, $\Sigma=\left\{i_{1}, i_{2}, \ldots i_{N}\right\}$, representing space-like infinities (cf. S. Dain, this volume). In principle, $\Sigma$ could consist of a union of such sets or of more complicated sets. In any of these situations there arises the question about the precise smoothness of the data 
near $\Sigma$ in suitably chosen coordinates.

It turns out that in general, even under the strongest smoothness assumptions on the 'free' data, there may occur terms of the form $r^{k} \log ^{l} r$ near $\Sigma$, where $r$ denotes the (non-physical) distance from $\Sigma$. However, it also turns out that under mild additional assumptions logarithmic terms will be absent near $\Sigma$. We will discuss some of the subtle questions arising here later.

\subsubsection{Gauge conditions, hyperbolicity, and local evolution}

In principle everything known about Einstein's equations also applies to our system. If we introduce a fictitious energy-momentum tensor by writing $\kappa T_{\mu \nu}=$ $2\left(L_{\mu \nu}-L g_{\mu \nu}\right)$ with a constant $\kappa \neq 0$ (introduced here to emphasize the similarity, we could set $\kappa=1$ ), our equations imply after a contraction of (27) the system

$$
\begin{gathered}
R_{\mu \nu}-\frac{1}{2} R g_{\mu \nu}=\kappa T_{\mu \nu} \\
\nabla_{[\lambda} T_{\rho] \nu}-\frac{1}{3} \nabla_{[\lambda} T g_{\rho] \nu}=\frac{1}{\kappa} \nabla_{\mu} \Omega d_{\nu \lambda \rho}^{\mu} \\
\nabla_{\mu} d^{\mu}{ }_{\nu \lambda \rho}=0 \\
\nabla_{\mu} \nabla_{\nu} \Omega=-\frac{\kappa}{2}\left(T_{\mu \nu}-\frac{1}{3} T g_{\mu \nu}\right) \Omega+s g_{\mu \nu} \\
\nabla_{\mu} s=-\frac{\kappa}{2}\left(T_{\mu \nu}-\frac{1}{3} T g_{\mu \nu}\right) \nabla^{\nu} \Omega
\end{gathered}
$$

which can be read as Einstein equations with certain matter fields, in which $R=-\kappa T$ represents a gauge source function. We note that in boundary value problems for these equations it has to be made sure, by the way the problems are posed and the data are given, that equation (27), which gives geometrical meaning to the fields $L_{\mu \nu}$ and $d^{\mu}{ }_{\nu \lambda \rho}$, will be satisfied.

Following the discussion of the Bianchi equation above (or that of [37], if one prefers to consider the metric instead of a frame field as a basic variable), it is not difficult to extract symmetric hyperbolic evolution equations, the reduced equations, from the last four subsystems. Of course, any choice here will leave us with constraint equations. It remains to discuss the first equation. In principle one can bring to bear here all the methods developed for the ADM equations if one feels most comfortable with these equations. However, it should be kept in mind that this is only one of the many possibilities of dealing with equation (27). In the references given in this article there can be found many others which have been employed successfully under various geometrical assumptions.

If one is dealing with the Einstein equation above or directly with equation (27), in either case one will have to make the decision whether to use the metric coefficients $g_{\mu \nu}$ or frame coefficients $e^{\mu}{ }_{k}$ as basic variables. Furthermore, one needs to choose gauge conditions, i.e. a way to specify the coordinates and, in the second case, the frame field. Usually this is done in terms of a gauge system of differential equations and suitable initial conditions. A large set of possibilities is known by now. 
Basic objectives in choosing the gauge are the following (cf. also 45]): (i) The gauge should be adapted to the type of problem (Cauchy problem, initialboundary value problem, characteristic initial value problem), (ii) The evolution equations should be simple, manifestly hyperbolic, or of some other form (e.g. 'elliptic-hyperbolic') which allows us to handle them in existence proofs or in numerical calculations (cf. the discussion by H.-O. Kreiss and O. Ortiz, this volume). There are available gauge condition which are governed by implicit wave equations like the harmonic gauge or the conditions for frame or spin frame fields employed in [27], [32], and there are known explicit conditions, in particular certain geometric gauge conditions, which lead to a reduction of the number of the unknowns (cf. [35], [37] and the discussion below) and which allow us to control to some extent the development of a degeneracy of the gauge. (iii) The gauge conditions as well as the constraints should be preserved during the evolution. (iv) The gauge should allow us to control its behaviour during the evolution in numerical or in analytical studies. (v) It should have a sufficiently long 'life time'.

While (ii) and (iii) are in the case of hyperbolic equations local properties which are accessible to direct calculations, (iv) and (v) depend very much on the given situation and cannot be handled in a general way. These points belong to the most difficult ones in the large scale evolution problem in numerical as well as in analytical studies.

There are known now various ways to perform hyperbolic reductions (cf. [37]) for initial value problems for the metric conformal field equations: given a suitable solution to the constraints, we find a reduced system of hyperbolic evolution equations for which the local existence and uniqueness of solutions follow from standard theorems, and, by invoking the available integrability conditions, we can derive a subsidiary system which allows us to show that all constraints and gauge conditions are preserved during the evolution if they are satisfied initially. The significance of the conformal field equations is determined to a large extent by the fact that they admit hyperbolic reductions irrespective of the sign of the conformal factor.

After we use the large freedom to choose the gauge system and the reduced system, the subsidiary system is essentially fixed. For the analytical discussions of solutions, the latter is only required to possess the uniqueness property, which ensures that trivial data imply the trivial solution. However, since in the numerical treatment of the Einstein equations the constraints are only satisfied up to a numerical error, the detailed propagation properties of the subsidiary system may become important in that context. A systematic analysis of how changes in the gauge system and the reduced system affect the propagation properties of the subsidiary system and whether this dependence can be used to choose reduced systems with improved evolution of the constraints has not been given yet. 


\subsubsection{The general conformal field equations}

We will now consider a system of conformal field equations which employs the additional freedom gained by admitting general Weyl connections. Combined with suitable gauge conditions it will lead to a system of evolution equations which is quite different from and in fact much simpler than the evolution systems obtained from the metric conformal field equations.

Assume that $\tilde{g}$ solves Einstein's vacuum field equations with cosmological constant $\lambda$ and let $g=\Theta^{2} \tilde{g}$ be given a conformal metric where $\Theta$ is some conformal factor. Furthermore, let $b$ be a smooth 1 -form. We denote by $\tilde{\nabla}$ and $\nabla$ the Levi-Civita connection of $\tilde{g}$ and $g$ respectively and by $\hat{\nabla}$ the Weyl connection for $\tilde{g}$ which satisfies $\hat{\nabla}-\tilde{\nabla}=S(b)$. It follows that $\hat{\nabla}=\nabla+S(f)$ with $f=b-\Theta^{-1} \nabla \Theta$. We define a further 1-form by setting $d_{\mu}=\Theta f_{\mu}+\nabla_{\mu} \Theta$.

Let $e_{k}$ be a frame field satisfying $g\left(e_{i}, e_{k}\right)=\eta_{i k}$. We denote by $\nabla_{k}$ and $\hat{\nabla}_{k}$ the covariant derivative in the direction of $e_{k}$ with respect to $\nabla$ and $\hat{\nabla}$ and define the connection coefficients $\hat{\Gamma}_{i}{ }^{j}{ }_{k}$ of $\hat{\nabla}$ in this frame field by $\hat{\nabla}_{i} e_{k}=\hat{\Gamma}_{i}{ }^{j}{ }_{k} e_{k}$. If we express now all tensor fields (except the $e_{k}$ ) as well as equations (30), (31), (26) and the condition that $\hat{\nabla}$ be torsion free in terms of the frame field and the connection coefficients, we get for the unknown

$$
u=\left(\begin{array}{llll}
e^{\mu} & \hat{\Gamma}_{i}{ }_{k}, & \hat{L}_{j k}, & d^{i}{ }_{j k l}
\end{array}\right),
$$

the system of equations

$$
\begin{gathered}
{\left[e_{p}, e_{q}\right]=\left(\hat{\Gamma}_{p}{ }^{l}{ }_{q}-\hat{\Gamma}_{q}{ }^{l}{ }_{p}\right) e_{l},} \\
e_{p}\left(\hat{\Gamma}_{q}{ }^{i}{ }_{j}\right)-e_{q}\left(\hat{\Gamma}_{p}{ }^{i}{ }_{j}\right)-\hat{\Gamma}_{k}{ }^{i}{ }_{j}\left(\hat{\Gamma}_{p}{ }^{k}{ }_{q}-\hat{\Gamma}_{q}{ }^{k}{ }_{p}\right)+\hat{\Gamma}_{p}{ }^{i}{ }_{k} \hat{\Gamma}_{q}{ }^{k}{ }_{j}-\hat{\Gamma}_{q}{ }^{i}{ }_{k} \hat{\Gamma}_{p}{ }^{k}{ }_{j} \\
=2\left\{g^{i}{ }_{[p} \hat{L}_{q] j}-g^{i}{ }_{j} \hat{L}_{[p q]}-g_{j[p} \hat{L}_{q]}{ }^{i}\right\}+\Theta d^{i}{ }_{j p q}, \\
\hat{\nabla}_{p} \hat{L}_{q j}-\hat{\nabla}_{q} \hat{L}_{p j}=d_{i} d^{i}{ }_{j p q}, \\
\nabla_{i} d^{i}{ }_{j k l}=0 .
\end{gathered}
$$

In the last equation the connection $\nabla$ is used. This poses no problem because the connection coefficients of $\nabla$ in the frame $e_{k}$ are related to those of $\hat{\nabla}$ by the purely algebraic relation $\hat{\Gamma}_{i}{ }^{j}{ }_{k}=\Gamma_{i}{ }^{j}{ }_{k}+\delta^{j}{ }_{i} f_{k}+\delta^{j}{ }_{k} f_{i}-\eta_{i k} \eta^{j l} f_{l}$, whence $f_{i}=1 / n \hat{\Gamma}_{i}^{k}{ }_{k}$.

No differential equations are given above for the fields $\Theta$ and $d_{k}=\Theta f_{k}+$ $\nabla_{k} \Theta$, they reflect the artificially introduced conformal gauge freedom. Note that they cannot be prescribed quite arbitrarily. For a solution which extends smoothly into a domain containing a set where $\Theta=0$, we should have $d=\nabla \Theta$ on this set.

To obtain the metric conformal field equations, (26), (27), (28) have been complemented by equations for the conformal factor which contain basic information on Einstein's equations. Finding a suitable gauge to obtain useful 
evolution equations was then considered a different issue. In the case of the equations above we will combine these two steps into one. The extended gauge freedom will be used here in a very special way. The question whether there are other useful possibilities has not been investigated.

We begin by describing the construction of a gauge. Let $\tilde{S}$ be a space-like hypersurface in the given vacuum solution $(\tilde{M}, \tilde{g})$. We choose on $\tilde{S}$ a positive 'conformal factor' $\Theta_{*}$, a frame field $e_{k *}$, and a 1-form $b_{*}$ such that $\tilde{g}\left(e_{i *}, e_{k *}\right)=$ $\Theta_{*}^{2} \eta_{i k}$ and $e_{0 *}$ is orthogonal to $\tilde{S}$. Then there exists through each point $x_{*} \in \tilde{S}$ a unique conformal geodesic $x(\tau), b(\tau)$ with $\tau=0$ on $\tilde{S}$ which satisfies there the initial conditions $\dot{x}=e_{0 *}, b=b_{*}$. These curves define a smooth caustic free congruence in a neighbourhood $U$ of $\tilde{S}$ if all data are smooth. Furthermore, $b$ defines a smooth 1 -form on $U$ which supplies a Weyl connection $\hat{\nabla}$ as described above. A smooth frame field $e_{k}$ and conformal factor $\Theta$ are then obtained on $U$ by solving (19), (20) for given initial conditions $e_{k}=e_{k *}, b=b_{*}$ on $\tilde{S}$. The frame field is orthonormal for the metric $g=\Theta^{2} \tilde{g}$, i.e. $g_{i k} \equiv g\left(e_{i}, e_{k}\right)=$ $\operatorname{diag}(1,-1,-1,-1)$. Dragging along local coordinates $x^{\alpha}, \alpha=1,2,3$ on $\tilde{S}$ with the congruence and setting $x^{0}=\tau$ we obtain a coordinate system. In this gauge we have on $U$

$$
\dot{x}=e_{0}=\partial_{\tau}, \quad \hat{\Gamma}_{0}{ }_{k}=0, \quad \hat{L}_{0 k}=0 .
$$

Coordinates, a frame field, and a conformal gauge as above will be referred to as a conformal Gauss system.

We could now derive from (20) and the conformal geodesics equations an equation for $d_{k}=\Theta b_{k}=\Theta f_{k}+\nabla_{k} \Theta$ and use it together with (20) to complement the system (39) to (42). Surprisingly, however, the fields $\Theta$ and $d_{k}$ can be determined explicitly (35): If $\tilde{g}$ is a solution to Einstein's vacuum equations with cosmological constant $\lambda$, the fields $\Theta$ and $d_{k}$ are given in our gauge by the explicit expressions

$$
\begin{gathered}
\Theta=\Theta_{*}\left(1+\tau<b_{*}, \dot{x}_{*}>+\frac{\tau^{2}}{2}\left(\Theta_{*}^{-2} \frac{\lambda}{6}+\frac{1}{2} g^{\sharp}\left(b_{*}, b_{*}\right)\right)\right), \\
d_{0}=\dot{\Theta}, \quad d_{a}=<b_{*}, \Theta_{*} e_{a *}>, \quad a=1,2,3,
\end{gathered}
$$

where the quantities with a subscript star are considered as constant along the conformal geodesics and $g^{\sharp}$ denotes the contravariant version of $g$.

With these expressions (39), (40), (41), (42) provide a complete system for $u$. In spite of the fact that we use a special gauge, we refer to these equations as the general conformal field equations to indicate that they employ the full gauge freedom preserving a conformal structure.

One might wonder why in the present case it should be sufficient to give the explicit expression for $\Theta$ and $b$ while in the case of the metric conformal field equation we had to use Einstein's equations again to obtain equations for the conformal factor. However, the occurrence of $\lambda$ shows that information about Einstein's equations is encoded in (44), (45). We note the following important consequence. If we have a sufficiently smooth solution to the metric conformal field equations in a domain comprising a non-empty set $\mathcal{J}=\{\Omega=0, d \Omega \neq 0\}$, 
it follows from (34) that $\nabla_{\rho} \Omega \nabla^{\rho} \Omega=-\lambda / 3$ on $\mathcal{J}$, i.e. the causal nature of $\mathcal{J}$ is determined by the field equations $([65])$. The analogous result

$$
\nabla_{k} \Theta \nabla^{k} \Theta=-\frac{1}{3} \lambda \quad \text { where } \quad \Theta=0,
$$

follows in the present case from (44), (45) and the definition of $b$ if the congruence does not degenerate where $\Theta$ vanishes ([35]).

Our gauge is not only distinguished by the fact that it is provided by the conformal structure itself and the explicit information on $\Theta$ and $b$, but also by a remarkable simplicity of the resulting evolution equations. Setting $p=0$ and observing the gauge conditions (43) we obtain from (39) - (42)

$$
\begin{gathered}
\partial_{\tau} e^{\mu}{ }_{q}=-\hat{\Gamma}_{q}{ }^{l}{ }_{0} e^{\mu}{ }_{l}, \\
\partial_{\tau} \hat{\Gamma}_{q}{ }^{i}{ }_{j}=-\hat{\Gamma}_{k}{ }^{i}{ }_{j} \hat{\Gamma}_{q}{ }^{k}{ }_{0}+g^{i}{ }_{0} \hat{L}_{q j}+g^{i}{ }_{j} \hat{L}_{0 q}-g_{j 0} \hat{L}_{q}{ }^{i}+\Theta d^{i}{ }_{j 0 q}, \\
\partial_{\tau} \hat{L}_{q j}=d_{i} d^{i}{ }_{j 0 q}, \\
\nabla_{i} d^{i}{ }_{j k l}=0 .
\end{gathered}
$$

Extracting from the Bianchi equation by the methods discussed above a symmetric hyperbolic system, we get symmetric hyperbolic reduced equations for those components of $u$ which are not determined explicitly by the gauge conditions. It has been shown in [35] for such a choice of reduced equations (assuming (44), (45)) that any solution which satisfies the constraints on a suitable spacelike hypersurface does indeed satisfy the complete set of field equations in the part of the domain of dependence of the initial data set where $\Theta$ is positive.

A system of similar simplicity is obtained if we use metric Gauss coordinates and the vacuum Bianchi identity to solve the vacuum field equations directly (24]). While it is well known, however, that in general Gauss coordinates tend to develop caustics quickly, it will be seen below that conformal Gauss coordinates can be arranged to cover large space-time domains or even entire maximal globally hyperbolic space-times in non-trivial cases.

Our main purpose in reformulating the field equations was to obtain results about their solutions. In fact, all the properties above have been used to establish properties of solutions by formulating and analyzing boundary value problems which take into account as far as possible the geometric nature of the situations under study. In the following we shall indicate the kind of questions which have been asked and the type of results obtained so far.

\section{The Penrose proposal}

Experience with other theories governed by hyperbolic field equations suggests that Einstein's theory should admit wave phenomena. For the same reason for which some work is required to bring to light the hyperbolic nature of Einstein's equation, we need to make an effort to exhibit the wave phenomena. Since the theory only supplies the metric and the associated curvature field, we need to 
explain which part of the gravitational field shows wave-like behaviour with respect to which reference structure and to what extent wave phenomena observed in mathematical expressions are of any physical significance. The standard textbook discussion of gravitational radiation bypasses this problem by postulating some ad hoc background solution and studying solutions to the linearized equations on it. This method allows one to apply the machinery developed in other areas of field theory, but specific features of Einstein's theory arising from its non-linearity are suppressed. Nowadays there exist much more sophisticated approximation methods, which also take into account insights from the approach to be described below (cf. [8], [78]).

Work initiated in the late 1950's by Pirani, Trautman, Bondi and others led to a concept of radiation which involves an 'approximation to nature', namely the idealization of an isolated system, but which does not require for its definition any mathematical approximations in the given framework. Clearly, the gravitational field can be expected to develop a wave-like character only sufficiently far away from the generating system. The idea then is to consider a gravitating system, consisting of stars, black holes, or bounded systems of stars like galaxies, which is so far away from other such systems that the latter can essentially be ignored and taken into account only in terms of the gravitational radiation emitted by them. Thus one is led to study the far field of this system.

Following a light ray which escapes from the system to infinity we may expect the field to become weaker and weaker and in fact asymptotically flat in the sense that the metric field approaches that of a limit Minkowski space. If some substructure, e.g. some component of the Weyl curvature, implies a non-trivial field on this limit space it might attain a natural interpretation as the radiation escaping from the system.

To convert this idea into a workable definition two things are needed: a precise description of the limit procedure and detailed information on the decay of the metric and the curvature field. Bondi et al. [9], Newman and Penrose [61], and Sachs [74] specify the limits in terms of so-called Bondi coordinates, which are generated by distinguished families of outgoing null hypersurfaces which extend in the future smoothly to infinity. On the hypersurfaces a convenient parameter is specified on the generating null geodesics which themselves are parameterized by spherical coordinates. This choice of coordinates is quite natural for analyzing radiation phenomena, since for hyperbolic field equations perturbations of the field essentially travel along characteristics.

The most delicate part of the whole project is to gain information on the decay. Obviously, we cannot prepare the systems of interest and let them go to observe the decay of the field. In fact, at present the main interest in our whole subject is to obtain information on wave forms mathematically to possibly help identify gravitational radiation in the signals supplied by the measuring devices. To prepare the systems and study their evolution for an infinite time by purely mathematical means was out of the question at a time when not even the Cauchy problem local in time was completely understood. Therefore the authors above had to rely on their experience with a few model examples, linearized theory, assumptions of simplicity, physical intuition etc. to make a reasonable guess. 
In [9], 74] the asymptotic behaviour was imposed by requiring that certain metric coefficients behave in Bondi coordinates similarly as in the case of Minkowski space while in [61] fall-off conditions for certain components of the Weyl curvature were specified. In both cases the assumptions proved consistent with the first few steps of a formal expansions involving the field equations. The authors could associate certain asymptotic data convincingly with the gravitational radiation field, and conclude from the behaviour of the Bondi-energymomentum that outgoing gravitational waves carry only positive energy ([9], [74).

Soon afterwards a new idea brought into the open the basic geometric structure underlying this analysis. In 65], [66 Penrose proposed to characterize the fall-off behaviour of gravitational fields in terms of the extensibility of the conformal structure through null infinity.

To illustrate the idea we begin by describing certain conformal embeddings of the simply connected, conformally flat standard solutions to the vacuum field equations $\operatorname{Ric}[\tilde{g}]=\lambda \tilde{g}$ into the Einstein cosmos (cf. also [52]). The latter is given by the manifold $\bar{M}=\mathbb{R} \times S^{3}$, endowed with the product line element $g=d s^{2}-d \omega^{2}$ where $d \omega^{2}=d \chi^{2}+\sin ^{2} \chi d \sigma^{2}$ denotes the standard line element on the 3-sphere with $d \sigma^{2}=d \theta^{2}+\sin ^{2} \theta d \phi^{2}$ the standard line element on the 2 -sphere. We shall in the following be mainly interested in the conformal extension of Minkowski space and shall use the other two examples only to illustrate certain arguments.

The case $\lambda=0$ : We express the metric of Minkowski space $\left(\tilde{M} \simeq \mathbb{R}^{4}, \tilde{g}=\right.$ $\left.\eta_{\mu \nu} d x^{\mu} d x^{\nu}\right)$ in spherical coordinates such that $\tilde{g}=d t^{2}-\left(d r^{2}+r^{2} d \sigma^{2}\right)$ and define a diffeomorphism $\Phi$ of $\tilde{M}$ onto the open subset $\Phi(\tilde{M})=\tilde{M}^{\prime} \equiv\{|s+\chi|<$ $\pi, \quad|s-\chi|<\pi, \quad \chi \geq 0\}$ of $\bar{M}$ such that $\Phi^{-1}$ is given by

$$
t=\frac{\sin s}{\cos s+\cos \chi}, \quad r=\frac{\sin \chi}{\cos s+\cos \chi} .
$$

It follows that $\Omega^{2} \Phi^{-1 *} \tilde{g}=g$ on $\tilde{M}^{\prime}$ with the conformal factor $\Omega=\cos s+\cos \chi$ which is positive on $\tilde{M}^{\prime}$. The embedded Minkowski space acquires then a conformal boundary $\mathcal{J}$ in $\bar{M}$, which is defined as the subset $\mathcal{J} \equiv \partial \tilde{M}^{\prime}=\mathcal{J}^{-} \cup \mathcal{J}^{+} \cup$ $\left\{i^{-}, i^{0}, i^{+}\right\}$, of $M$, where the hypersurfaces $\mathcal{J}^{ \pm}=\{\tau \pm \chi= \pm \pi, 0<\chi<\pi\}$, which can be thought of as being generated by endpoints of null geodesics, represent future resp. past null infinity, the points $i^{ \pm}=\{\chi=0, \tau= \pm \pi\}$ represent future resp. past time-like infinity and the point $i^{0}=\{\chi=\pi, \tau=0\}$ represents space-like infinity. The key point here is that the embedded Minkowski space together with its conformal structure and the conformal factor $\Omega$ can be extended smoothly to the manifold with boundary $M=\tilde{M}^{\prime} \cup \mathcal{J}^{+} \cup \mathcal{J}^{-}$such that $\Omega=0, d \Omega \neq 0$ on the null hypersurfaces $\mathcal{J}^{ \pm} \simeq \mathbb{R} \times S^{2}$.

It may be of interest to note that $\mathrm{H}$. Weyl already in 1923 considers the conformal embedding of Minkowski space into a quadric in the five dimensional real projective space in a relativity inspired discussion of the Möbius group and refers to the set which corresponds to $\mathcal{J}$ as to the 'sphere at infinity' ([80]). The fact that Weyl did not see, in spite of its beautifully geometric nature, the 
generalization proposed in 65, indicates to what extent the kind of questions being asked and, in fact, our whole view of the subject have changed since then.

The case $\lambda<0$ : The de Sitter space is given by $\left(\tilde{M}=\mathbb{R} \times S^{3}, \tilde{g}=d t^{2}-\right.$ $\left.\cosh ^{2} t d \omega^{2}\right)$. The map $\tilde{M} \ni(t, \vartheta) \stackrel{\Phi}{\rightarrow}\left(s=\arctan e^{t}-\frac{\pi}{4}, \vartheta\right) \in \bar{M}$, where $\vartheta$ denotes the points in $S^{3}$, maps de Sitter space diffeomorphically onto the open subset $\tilde{M}^{\prime}=[-\pi / 4, \pi / 4] \times S^{3}$ of the Einstein cosmos. It satisfies $\Omega^{2} \Phi^{-1 *} \tilde{g}=g$ on $\tilde{M}^{\prime}$ with the positive conformal factor $\Omega=\cos (2 s)$. Again the embedded space together with its conformal structure and the conformal factor $\Omega$ can be extended smoothly to a manifold with boundary $M=\tilde{M}^{\prime} \cup \mathcal{J}^{+} \cup \mathcal{J}^{-}$such that $\Omega=0, d \Omega \neq 0$ on the space-like hypersurfaces $\mathcal{J}^{ \pm}=\{ \pm \pi / 4\} \times S^{3}$ of $M$.

The case $\lambda>0$. In spherical coordinates we obtain anti-de Sitter covering space in the form $\left(\tilde{M}=\mathbb{R}^{4}, \tilde{g}=\cosh ^{2} r d t^{2}-d r^{2}-\sinh ^{2} r d \sigma^{2}\right)$. The map $\tilde{M} \ni(t, r, \theta, \phi) \stackrel{\Phi}{\rightarrow}\left(s=t, \chi=2 \arctan \left(e^{r}\right)-\frac{\pi}{2}, \theta, \phi\right) \in \bar{M}$ defines a diffeomorphism of $\tilde{M}$ onto the subset $\tilde{M}^{\prime}=\{\chi<\pi / 2\}$ of the Einstein cosmos which satisfies $\Omega^{2} \Phi^{-1 *} \tilde{g}=g$ on $\tilde{M}^{\prime}$ with the positive conformal factor $\Omega=\cos \chi$. The embedded space together with its conformal structure and the conformal factor $\Omega$ can be extended smoothly to a manifold with boundary $M=\tilde{M}^{\prime} \cup \mathcal{J}$ such that $\Omega=0, d \Omega \neq 0$ on the time-like hypersurface $\mathcal{J}=\{\chi=\pi / 2\} \simeq \mathbb{R} \times S^{2}$ of $M$.

The conformal extensions above are of interest already because they provide detailed information about the global causal relations in the three space-times and allow us to discuss the asymptotic behaviour of solutions to conformally invariant equations such as the Maxwell equations easily and with any desired precision. For our present discussion they are of particular interest because the three conformally embedded spaces and the given conformal factors provide solutions of the metric conformal field equations which extend, as solutions, through the conformal boundaries. The question whether this fact can be considered as a model for the behaviour of more general gravitational fields leads us (in hindsight) to the generalization proposed in 65 .

The embedding formulae given above are distinguished by their simplicity but may look otherwise somewhat arbitrary. It turns out that we do not have much freedom. For given $\vartheta \in S^{3}$ the curve $\mathbb{R} \ni \tau \rightarrow\left(s=2 \arctan \frac{\tau}{2}, \vartheta\right) \in \bar{M}$ is a conformal geodesic on the Einstein cosmos. If $\chi(\vartheta) \neq 0, \pi$, this curve intersects the conformal boundary $\mathcal{J}^{ \pm}$of the embedded Minkowski space for values $\tau_{ \pm}(\vartheta) \neq 0$ with $\tau_{-}=-\tau_{+}$. By specifying suitable initial data on the hypersurface $\{t=0\}$, this curve can be constructed completely in terms of Minkowski space and we find that the conformal factor $\Theta$ obtained by solving (20) along this conformal geodesic remains positive on Minkowski space and vanishes precisely as $\tau \rightarrow \tau_{ \pm}$, where $\dot{\Theta}\left(\tau_{ \pm}\right) \neq 0$. It follows that, apart from a coordinate transformation and a rescaling with a conformal factor $\omega$ which is positive on $\bar{M}$, the conformal extension can be constructed completely by integrating a suitable congruence of conformal geodesics and an associated conformal factor on Minkowski space and extending the resulting formulae by continuity (cf. 411 for details).

We could have surmised the possibility for this already when we encountered 
formula (44). It shows that for suitable initial data $\Theta$ has precisely two zeros. This also raises a question. Does the fact that the formula is valid on a general vacuum solutions suggest that a similar procedure works under much more general assumptions? We are thus led again to the generalization proposed in 65.

Stating in general terms what has been found above, one arrives at the following definition.

Definition 3.1 A smooth space-time $(\tilde{M}, \tilde{g})$ is called asymptotically simple if there exists a smooth, oriented, time-oriented, causal space-time $(M, g)$ and on $M$ a smooth function $\Omega$ such that:

(i) $M$ is a manifold with boundary $\mathcal{J}$,

(ii) $\Omega>0$ on $M \backslash \mathcal{J}$ and $\Omega=0, d \Omega \neq 0$ on $\mathcal{J}$,

(iii) there exists an embedding $\Phi$ of $\tilde{M}$ onto $\Phi(\tilde{M})=M \backslash \mathcal{J}$ such that $\Omega^{2} \Phi^{-1 *} \tilde{g}=$ $g$.

(iv) each null geodesic of $(\tilde{M}, \tilde{g})$ acquires two distinct endpoints on $\mathcal{J}$.

This definition, which only involves the conformal structure of $(M, g)$, reflects the differential geometric aspects of the situations considered above. It implies restrictions on the global structure of the space-time. Condition $(i v)$, for instance, implies that all null geodesics are complete. The set $\mathcal{J}$ thus represents a boundary at null infinity which can be thought of as being generated by ideal endpoints of null geodesics. Conditions $(i),(i i)$ are somewhat redundant, since (ii) implies already that $\mathcal{J}$ is a smooth hypersurface. However, this is not the only purpose of $(i i)$. Together with $(i i i)$ it specifies how precisely $\Phi^{-1 *} \tilde{g}$ is to be rescaled to obtain a smooth, non-degenerate metric. The definition above was motivated by the following new idea (65).

Penrose proposal: Far fields of isolated systems behave like asymptotically simple space-times in the sense that they can be smoothly extended to null infinity, as indicated above, after suitable conformal rescalings.

It should be noted that we have only tried to indicate the idea here. It should be applied with understanding and the definition above may require modifications in certain situations. For instance, in applying the definition to physically realistic situations it may be advisable to replace $(i v)$ by some other completeness conditions (cf. [50] for an example) to allow for the possibility that some of the null geodesics enter black holes. The standard example here is the Schwarzschild-Kruskal solution, which has two asymptotic ends which admit smooth conformal extensions (cf. B. Schmidt, this volume). We consider any such changes as minor adaptations. As we shall see below, there may be required much more subtle ones.

While in the earlier description of fall-off conditions coordinates play a crucial role and while even in case of asymptotically simple space-times it is often convenient to use Bondi coordinates, the present characterization is completely independent of any distinguished coordinate systems and therefore much more flexible than the earlier ones. But the beauty of the proposal is that it brings out clearly the key geometrical structure. 
Central and critical is the requirement (iii) and the smoothness assumption. The smoothness properties of the rescaled field $g$ are an expression for the falloff behaviour of the physical field $\tilde{g}$. In view of the Penrose proposal definition (3.1) acquires suddenly a much stronger interest. It is now in competition with another requirement and involves an extremely sharp characterization of the decay of fields which are governed by the quasi-linear, gauge hyperbolic field equations.

The question whether the assumption that the field equations hold near $\mathcal{J}$ is consistent with the smoothness and conditions $(i)-($ iii $)$, or whether the smoothness assumptions made in definition (3.1) need to be relaxed has been and, as seen below, still is a matter of debate. The question is not whether $C^{\infty}$ should be replaced by $C^{k}$ for some large $k$. The question is whether solutions to the field equations admit conformal extensions of class $C^{k}$ where $k$ can be chosen large enough to make the proposal meaningful and, related to this, whether the conformal Weyl tensor tends to zero on the conformal boundary.

Penrose addresses this problem in [66]. For later comparison we recall the assumptions and give (a version of) the main argument, using the spin frame formalism. Consider a smooth solution to the metric conformal field equations with $\lambda=0$ on the physical manifold $\tilde{M}$ and assume that it admits a conformal extension to a manifold $M=\tilde{M} \cup \mathcal{J}^{+}$of class $C^{4}$ such that $g$ and $\Omega$ extend to fields in $C^{3}(M)$. The conformal Weyl spinor $\Psi_{a b c d}$ is then in $C^{1}(M)$.

By definition, $\Omega=0, d \Omega \neq 0$ and, as observed earlier, $\nabla_{a a^{\prime}} \Omega \nabla^{a a^{\prime}} \Omega=0$ on $\mathcal{J}^{+}$. We assume that there is a space-like surface $\Sigma$ in $\mathcal{J}^{+}$which intersects each null generator of $\mathcal{J}^{+}$exactly once. In a neighbourhood of $\Sigma$ there are then $C^{3}$ spinor fields $o^{a}, \iota^{a}$ with $\iota^{a} o_{a}=1$ such that $\nabla^{a a^{\prime}} \Omega=-o^{a} \bar{o}^{a^{\prime}}$ on $\mathcal{J}^{+}$ and the complex vector field $m^{a a^{\prime}}=o^{a} \bar{\iota}^{a^{\prime}}$ is tangent to $\Sigma$. Note that the set of such spin frames defines a reduction $U(\Sigma)$ of the bundle of normalized spin bases over $\Sigma$ to the subgroup $U(1)$ of $S L(2, \mathbb{C})$, where $e^{i \frac{\theta}{2}} \in U(1)$ acts on the spin frames by $(o, \iota) \rightarrow\left(e^{i \frac{\theta}{2}} o, e^{-i \frac{\theta}{2}} \iota\right)$. A $2: 1$ bundle morphism maps $U(\Sigma)$ onto the bundle $O^{+}(\Sigma)$ of oriented frames which are orthonormal for the induced metric $h$ on $\Sigma$ such that we can write $m=1 / \sqrt{2}\left(r_{1}+i r_{2}\right)$ with $r \equiv\left\{r_{A}\right\}_{A=1,2} \in O^{+}(\Sigma)$. Denote by $u$ the generator of the structure group $S O(2)$ of $O^{+}(\Sigma)$ with components $u^{A}{ }_{B}=\delta^{A}{ }_{1} \delta^{2}{ }_{B}-\delta^{A}{ }_{2} \delta^{1}{ }_{B}$ and by $Z$ the vertical vector field on $O^{+}(\Sigma)$ generated by $u$.

Equation (32) reduces on $\mathcal{J}^{+}$to $\nabla_{a a^{\prime}} \nabla_{b b^{\prime}} \Omega=s \epsilon_{a b} \epsilon_{a^{\prime} b^{\prime}}$. Since $o^{a} \nabla_{a a^{\prime}}$ is an interior operator on $\mathcal{J}^{+}$it follows that $o^{a} \nabla_{a a^{\prime}}\left(o_{b} \bar{o}_{b^{\prime}}\right)=-s o_{b} \epsilon_{a^{\prime} b^{\prime}}$ on $\mathcal{J}^{+}$ from which we obtain by suitable contractions $\iota^{a} \nabla_{m} o_{a}+\bar{\iota}^{a^{\prime}} \nabla_{m} \bar{o}_{a^{\prime}}$ whence $\gamma \equiv g\left(\nabla_{m} m, \bar{m}\right)=-2 \iota^{a} \nabla_{m} o_{a}$. Given $\gamma$, the connection $\mathcal{D}$ of $h$ is known, because $\mathcal{D}_{m} m=-\gamma m, \mathcal{D}_{m} \bar{m}=\gamma \bar{m}$.

With $\phi_{a b c d}=\Omega^{-1} \Psi_{a b c d}$ the Bianchi equation (36) takes on $\tilde{M}$ the form

$$
\nabla_{a^{\prime}}^{a} \Omega \Psi_{a b c d}-\Omega \nabla_{a^{\prime}}^{a} \Psi_{a b c d}=0 .
$$

(We note that in the case where $0 \neq \lambda=-3 \nabla_{f f} \Omega \nabla^{f f^{\prime}} \Omega$ on $\mathcal{J}$ this implies at each point of $\mathcal{J}$ immediately that $0=-3 \nabla_{e a^{\prime}} \Omega \nabla^{a a^{\prime}} \Omega \Psi_{a b c d}=\lambda \Psi_{e b c d}$.) It follows that $\Psi_{a b c d}=\alpha o_{a} o_{b} o_{c} o_{d}$ on $\mathcal{J}^{+}$with $\alpha=\Psi_{a b c d} \iota^{a} \iota^{b} \iota^{c} \iota^{d}$. If in addition 
it is assumed that

$$
\Omega \nabla_{e e^{\prime}} \nabla^{a}{ }_{a^{\prime}} \Psi_{a b c d} \rightarrow 0 \quad \text { at } \quad \mathcal{J}^{+},
$$

then equation (47) implies, after taking a covariant derivative, symmetrizing in $a^{\prime}$ and $e^{\prime}$, and performing some spinor algebra, the equation $o^{e} \nabla_{e e^{\prime}} \Psi_{a b c d}=0$ on $\mathcal{J}^{+}$. With the special form of the conformal Weyl spinor on $\mathcal{J}^{+}$it follows after contraction with $b^{b^{\prime}}$

$$
\nabla_{m} \alpha-2 \gamma \alpha=0 \quad \text { on } \quad \mathcal{J}^{+} .
$$

By its definition the $C^{1}$ function $\alpha$ can be considered as a 0 -form on $O^{+}(\Sigma)$ of spin weight -2 , i.e.

$$
Z \alpha=-2 i \alpha \quad \text { on } O^{+}(\Sigma) .
$$

Equation (49) says that the covariant differential of $\alpha$ on $O^{+}(\Sigma)$ (cf. [21]) vanishes on vector fields which project onto $m$. It can thus be expressed in the form

$$
\left(H_{1}+i H_{2}\right) \alpha=0 \quad \text { on } O^{+}(\Sigma),
$$

where for $A=1,2$ we denote by $H_{A}$ the horizontal vector field on $O^{+}(\Sigma)$ which projects at the point $r \in O^{+}(\Sigma)$ onto the tangent vector $r_{A}$ of $\Sigma$.

Suppose now that $\Sigma$ is diffeomorphic to the two-sphere $S^{2}$. Because $S^{2}$ carries only one conformal structure the conformal scaling of $g$ can be chosen near $\Sigma$ such that $(\Sigma, h)$ can be identified isometrically with $\left(S^{2},-d \sigma^{2}\right)$. This allows us to identify $O^{+}(\Sigma)$ with $S O(3)$, the isometry group of $S^{2}$, such that there are left invariant vector fields $Y_{i}, i=1,2,3$, on $S O(3)$ which satisfy under this identification $Y_{1}=H_{2}, Y_{2}=H_{1}, Y_{3}=Z$ and $\left[Y_{i}, Y_{j}\right]=\epsilon_{i j k} Y_{k}$, where $\epsilon_{i j k}$ is totally anti-symmetric with $\epsilon_{123}=1$. Equations (50), (51) then imply the distributional equation

$$
-\frac{1}{2}\left(Y_{1}^{2}+Y_{2}^{2}+Y_{3}^{2}\right) \alpha=\alpha \quad \text { on } \quad O^{+}(\Sigma) .
$$

Since the operator on the left hand side is the Casimir operator with respect to the Killing form on $S O(3)$, the solution space of this equation is well known (cf. [76]). It is spanned by the matrix elements of an irreducible three-dimensional unitary representation of the group $S U(2)$ (in the notation of [38] the functions $T_{2}{ }^{i}{ }_{j}$ ), which descends to $S O(3)$. Because the only function in this space which satisfies (50) is $\alpha=0$, it follows that the Weyl spinor field vanishes on $\Sigma$. Thus, $\phi_{a b c d}$ extends in a continuous fashion to $\mathcal{J}^{+}$and, as a consequence, satisfies Sachs' peeling property ([66], [73], [74]).

Remarkable as it is that such a conclusion can be drawn from the special conformal covariance of the Bianchi equations, the fact that we are dealing with a spin-2 field, and the topological assumption on $\Sigma$, there remains the question whether it follows from the nature of the long time evolution by the field equations that the smoothness assumptions made above and (48) can be satisfied and whether this is in fact the case for a sufficiently large class of solutions. 
There are two different issues here. We start with a discussion of the situation where the vacuum field equations with a cosmological constant $\lambda$ are satisfied locally near a point $p$ of $\mathcal{J}$ and where $\Omega$ and $g$ are smooth. As seen earlier, the conformal field equations then imply $\nabla_{\rho} \Omega \nabla^{\rho} \Omega=-\lambda / 3$. In particular, $\mathcal{J}$ is a null hypersurface if $\lambda=0$. In that case we can expect $\mathcal{J}$ to have two components $\mathcal{J}^{ \pm}$and $p \in \mathcal{J}^{+}$. To get some idea of the class of solutions which are compatible with conditions $(i)-($ iii $)$, we consider the asymptotic characteristic initial value problem for the metric conformal field equations where data are prescribed on two intersecting hypersurfaces which are to assume, by the arrangement of the initial data on them, the meaning of an outgoing null hypersurface $\mathcal{N}$ which meets $\mathcal{J}^{+}$in a space-like 2-surface $\Sigma$ and the part $\mathcal{J}_{*}^{+}$of $\mathcal{J}^{+}$in the past of $\Sigma$. This is essentially the problem underlying the formal expansions studied in [9], [61], [74].

All solutions for which the rescaled conformal Weyl tensor $d^{i}{ }_{j k l}$ has a smooth limit on $\mathcal{J}_{*}^{+}$can be characterized in the analytical as well as in the $C^{\infty}$ case (25], [57]) in the sense that for suitably given data the existence of a unique local solution near $\Sigma$ can be shown. The freedom to prescribe data on $\mathcal{N}$ and $\mathcal{J}_{*}^{+}$, which are in each case two components of $d^{i}{ }_{j k l}$ referred to as the null data, turns out to be the same as in the standard characteristic initial value problem for Einstein's vacuum field equations (corresponding to the special geometric nature of the hypersurface $\mathcal{J}_{*}^{+}$, there is a slight difference with the latter problem concerning the data which may be prescribed in addition to the null data on the intersection of the two null hypersurfaces). If, as above, the tensor $d^{i}{ }_{j k l}$ is represented by the symmetric spinor $\phi_{a b c d}$, then $\phi_{a b c d} \iota^{a} \iota^{b} \iota^{c} \iota^{d}$ and $\phi_{a b c d} o^{a} o^{b} o^{c} o^{d}$ denote the null data on $\mathcal{N}$ and $\mathcal{J}^{+}$respectively. It is quite a remarkable property of the conformal field equations that they define the conformal boundary $\{\Omega=0\}$ in terms of one of their unknowns without restricting the freedom to specify smooth data there.

The null data $\phi_{a b c d} o^{a} o^{b} o^{c} o^{d}$ on $\mathcal{J}^{+}$have a natural interpretation as the outgoing radiation field. Further important physical concepts can be associated with the hypersurface $\mathcal{J}^{+}$or subsets of it and the questions of interpretation have been extensively analyzed (cf. also [5], 49]). All indications suggest that we are on the right track.

The situation is similar in the de Sitter-type case $\lambda<0$. Here null infinity is space-like. We ask whether we can characterize the solutions which admit a smooth conformal extension at past null infinity $\mathcal{J}^{-}$under the (natural) assumption that this hypersurface is orientable and compact as in the case of de Sitter space and the further assumption that $d^{i}{ }_{j k l}$ extends smoothly to $\mathcal{J}^{-}$. Again, for suitably given data on $\mathcal{J}^{-}$there can be shown the existence of a unique solution to the conformal field equations. The freedom to specify data on $\mathcal{J}^{-}$is essentially the same as in a standard Cauchy problem with the exception that the mean intrinsic curvature of the initial hypersurface cannot be prescribed (29]). This appears natural, because $\mathcal{J}^{-}$is a very particular hypersurface with respect to the solution. Another peculiar feature consists in the fact that the Hamiltonian constraint becomes trivial on $\mathcal{J}^{-}$.

The situation where $\lambda>0$, in which case null infinity is time-like, differs 
in various aspects from the other cases. In this case the assumption of the smoothness of this limit has a different status than in the other cases. It appears that in general there would not even exist complete null geodesics if the field $d^{i}{ }_{j k l}$ tended to blow up sufficiently fast 'far out'. However, there is available a detailed discussion of the existence of solutions for which $d^{i}{ }_{j k l}$ has a smooth limit to $\mathcal{J}([35])$.

The solutions studied in these three situations are semi-global in the sense that in terms of the physical metric their domains are of infinite extent and even comprise asymptotic regions. However, this is not sufficient to answer the question posed above. Here comes in the second issue concerning the compatibility of asymptotic simplicity with the field equations. What we need to control is the behaviour of the solutions as they evolve towards null infinity. Thus, we cannot assume anything about the field $d^{i}{ }_{j k l}$ (except in the anti-de Sitter-type solutions where boundary data must be given at space-like and null infinity to characterize the solutions uniquely) and it is not clear a priori whether it remains bounded near null infinity. We need to find out whether it behaves, together with the other unknowns in the conformal field equations, such that a conformal extension $(M, g)$ of sufficient smoothness can be constructed.

There exists a model case which illustrates the type of result we would like to have. In the case of the de Sitter space considered above $(\lambda=-3)$ we consider the hypersurface $S=\{t=0\}$. The Cauchy data induced by de Sitter space on $S$ are given by the standard metric on $S^{3}$ and a vanishing extrinsic curvature. It turns out that data for the equation $\operatorname{Ric}[\tilde{g}]=\lambda \tilde{g}$ which are sufficiently close to the de Sitter data evolve into solutions which are asymptotically simple in the sense of definition (3.1), or, in other words, asymptotic simplicity is non-linearly stable ([30], [32]). Here $d^{i}{ }_{j k l}$ attains a smooth limit at the conformal boundary as a consequence of the evolution process and the assumptions on the initial data. The fact that the Schwarzschild-de Sitter solution has time slices diffeomorphic to $S^{2} \times S^{1}$ but develops horizons, singularities, and only patches of a smooth boundary shows that neither the compactness of the initial hypersurface nor the sign of the cosmological constant is sufficient for this behaviour. Remarkably, it has been shown recently that a solution with a compact Cauchy hypersurface can admit smooth conformal boundaries in the past as well as in the future only if the Cauchy hypersurface satisfies the topological restriction that its fundamental group be finite (柾, cf. G. Galloway, this volume, for further results concerning de Sitter-type solutions).

An analogous stability result in the Minkowski-type case $\lambda=0$ is still lacking. We shall see that a result of this simplicity cannot be expected.

\section{Asymptotic behaviour of vacuum fields with vanishing cosmological constant}

For physical applications one would like to know how the sources interact with each other and with the gravitational field and to what extent properties of 
the far field reflect this interaction. For a first mathematical analysis of the general behaviour of the far fields details of the sources are irrelevant and it is natural to ignore them in a first study. Thus we are led to ask whether there exist other solutions to the Einstein equations $\operatorname{Ric}[\tilde{g}]=0$ than Minkowski space which satisfy the requirements of definition (3.1).

Two different types of initial value problems have been studied in this context. The most important one is the standard Cauchy problem where Cauchy data are prescribed on a space-like hypersurface $S$ with one or several asymptotically Euclidean ends. If there are several such ends we can expect black holes to develop and condition (iv) to be violated. Therefore, and because it is the evolution near an end we want to focus on, we will concentrate on the case $S \simeq \mathbb{R}^{3}$ with one end. Standard examples are the hypersurface $\{t=0\}$ in Minkowski space with the induced data or perturbations thereof.

The other type of problem is the hyperboloidal initial value problem where data are prescribed on a hypersurface $H$ with boundary $\Sigma$, which is thought of as a hypersurface in an asymptotically simple space-time which extends to future null infinity $\mathcal{J}^{+}$, intersects $\mathcal{J}^{+}$in $\Sigma$, and is space-like everywhere. The boundary $\Sigma$ can have several components, examples exist in the Schwarzschild-Kruskal space-time (cf. B. Schmidt, this volume). Again we will concentrate on the case where only one asymptotic region exists such that $H \simeq\left\{x \in \mathbb{R}^{3}|| x \mid \leq 1\right\}$ and $\Sigma \simeq S^{2}$. The standard example of such a hypersurface is given by the extension of the space-like hyperboloid $\left\{t^{2}-|x|^{2}=1, t>0\right\}$ in Minkowski space to $\mathcal{J}^{+}$. Its interior inherits from Minkowski space a metric of constant negative curvature which can be rescaled to become Euclidean.

While the hyperboloidal initial value problem has been introduced initially as an intermediate step towards a treatment of the standard Cauchy problem (26]), it has gained in recent years an interest in its own as a basis for numerical calculations (cf. J. Frauendiener and S. Husa, this volume). It is intrinsically non-time-symmetric. The future evolution will extend under suitable assumptions to null infinity while the past time evolution develops a Cauchy horizon which corresponds to a part $N$ of an outgoing wave front. The domain covered by its evolution coincides with that which can be determined by the characteristic initial value problem where data are prescribed on $N$ (if this set is sufficiently smooth).

\subsection{The hyperboloidal initial value problem}

Since, for reasons which will become clear later, the hyperboloidal initial value problem is much easier to analyze in the context of asymptotic simplicity than the standard Cauchy problem, it has been analyzed much earlier and much more is known about it. If one wants to construct its solutions by solving the conformal field equations, the first task is to provide conformal initial data. In the analyses available so far, in which a non-vanishing constant mean extrinsic curvature is assumed, certain data can be prescribed on $H$ arbitrarily, certain

data are obtained by solving elliptic equations which degenerate on $\Sigma$, and the remaining data are obtained by differentiation and algebraic operations which 
involve divisions by the conformal factor, which vanishes on $\Sigma$. We give an outline of the results needed in the following discussion (for further information cf. L. Andersson, this volume).

The subtleties arise at $\Sigma$. In [1, [3] free data are prescribed which extend smoothly to $\Sigma$. It turns out that (a) in general the remaining data have expansions in terms of powers of $r$ and $\log r$, where $r$ denotes the conformal distance from $\Sigma$ (i.e. they admit polylogarithmic expansions at $\Sigma$ ), while (b) all data will be smooth if the free data satisfy certain regularity conditions which require that a few functions derived from the free data vanish on $\Sigma$. A much more general class of data is considered in [2]. In particular, (c) free data are prescribed which admit polylogarithmic expansions at $\Sigma$ and it is shown that the remaining data also admit such expansions.

The conformal field equations have been used to study the evolution of some of these data. In the case (b) the data evolve into solutions which satisfy the first three conditions of definition (3.1) and which admit in fact a conformally regular point $i^{+}$at time-like infinity, analogous to the point $i^{+}$in the case of Minkowski space, if the data are sufficiently close to Minkowskian hyperboloidal data ([26]), [30], [32]). Recently also the existence of solutions to the conformal field equations for data of the type (a) has been shown on a manifold which contains a boundary hypersurface $\mathcal{J}^{\prime}$ on which the unknown $\Omega$ of the conformal field equations vanishes. The solution is 'rough' at $\mathcal{J}^{\prime}$ and it is expected that it admits a polyhomogeneous expansion there ([60], cf. also [14]). The evolution of data of type (c) has not been studied yet.

While the basic idea underlying the Penrose proposal, namely to characterize the asymptotic behaviour of solutions in terms of conformal rescalings, is still being used in this study of asymptotically rough data by employing the conformal field equations, this situation certainly forbids any straightforward application of definition (3.1). The result seems to indicate that the smoothness assumption in definition (3.1) may be too strong. However, before we can arrive at a conclusion here we need to answer the following question: of which physical relevance are data of type (a) and (c), are they needed to model the systems of interest and which are the 'systems of interest'?

A clear answer could be given, if it could be shown that standard Cauchy data by necessity develop into solutions containing hyperboloidal data of type (a) or (c). The results for data of type (b) tell us that the decision about the smoothness of the conformal structure near null infinity is essentially made near space-like infinity. Thus we need to analyze the behaviour of the solution to the standard Cauchy problem there.

\subsection{On the existence of asymptotically simple vacuum so- lutions}

We shall see below that the general analysis of the conformal structure of solutions to the Einstein equations near space-like infinity is quite complicated. On the other hand, static or, more generally, stationary asymptotically flat vacuum solutions are known to admit smooth conformal extensions through null infinity 
(cf. [18]). Thus, to avoid the difficulties discussed below one could try to construct asymptotically flat standard Cauchy data (on $\mathbb{R}^{3}$ say) which agree with stationary data near space-like infinity. The time evolution of these data would then be stationary near space-like infinity and clearly contain smooth hyperboloidal hypersurfaces. One could then hope to combine this with the results of [30] to show the existence of solutions to the Einstein equations which satisfy all conditions of definition (3.1).

The first attempt at this was made by Cutler and Wald who succeeded in constructing smooth initial data for the Einstein-Maxwell equations with arbitrarily small ADM mass which agree with Schwarzschild data near spacelike infinity $(17])$. Using the results of [32, they were able to show for the first time the existence of smooth solutions to the Einstein-Maxwell equations which satisfy the conditions of asymptotic simplicity. The vacuum case remained difficult.

More recently Corvino developed a gluing construction by which an arbitrary smooth, time-symmetric, asymptotically flat solution $\left(\mathbb{R}^{3}, h\right)$ to the constraints can be deformed on a certain annulus $\left\{x \in \mathbb{R}^{3}|0<R<| x \mid<2 R\right\}$, such that it extends with a given finite differentiability to Schwarzschild data outside $2 R$ while remaining unchanged inside the radius $R$ and still satisfying the constraints everywhere ([16]). It turned out difficult, however, to keep the radius $R$ fixed or to control its growth sufficiently if the construction is applied to families of initial data for which the ADM mass $m$ is going to zero. This makes it impossible to obtain the desired result by an immediate application of [30].

Quite recently Chruściel and Delay obtained a similar result based on a modification of Corvino's technique in which the radius $R$ as well as the differentiability class $C^{k}$ may be kept fixed while $m \rightarrow 0$ if they base their construction on initial data $\left(\mathbb{R}^{3}, h\right)$ such that in the standard coordinates on $\mathbb{R}^{3}$ the parity condition $h(x)=h(-x)$ is satisfied by the 3-metric ([15]). In this case it is easy to construct in a systematic way hyperboloidal hypersurfaces in the time evolution of the data which contain the part $\left\{x \in \mathbb{R}^{3}|| x \mid<2 R\right\}$ of the initial Cauchy hypersurface as a subset and which extend into to future of the initial hypersurface in the Schwarzschild part of the evolution. It can be arranged that the induced data approach Minkowskian hyperboloidal data if $h$ approaches the Euclidean metric on $\mathbb{R}^{3}$ (cf. 41 for a construction based on conformal Gauss systems and 38 for the discussion of a smooth limit of Schwarzschild solutions to the Minkowski solution near space-like infinity). Since this can be done with sufficient smoothness, the results of [30] and the fact that the data are time-symmetric then imply the existence of non-trivial solutions to the vacuum field equations which satisfy the conditions of definition (3.1) with a finite differentiability of the rescaled metric which can be chosen to be large. For these solutions the rescaled conformal Weyl tensor is differentiable on the conformally extended space and there exist conformal extensions containing regular points $i^{ \pm}$ which represent future and past time-like infinity. This resolves a question which remained open and controversial for forty years.

While it is reassuring to see that we are not discussing a class of solutions which consists only of Minkowski space, it is clear that the result does not 
answer all our questions. The solutions obtained here are very special. Being Schwarzschildean near space-like infinity, their NP constants vanish ( [42], [62]). As a consequence the rescaled conformal Weyl tensor $d^{i}{ }_{j k l}$ vanishes at $i^{ \pm}$(《46], [63]). However, from the numerical calculation of solutions with a regular $i^{+}$ we know that in general $d^{i}{ }_{j k l}$ does not vanish at $i^{+}(\sqrt[54]{)})$. This suggests that modifying initial data sets to become Schwarzschild near infinity considerably reduces their radiation content.

Hopefully, there will be developed generalizations of the method which will allow us to glue other stationary data to given data such that we prescribe multipoles moments and non-vanishing NP constants. Then the argument above will not be available any longer and the data will gain in interest in the modeling of physical systems.

But this will still not help us answer the decisive question: what degree of restriction is implied by 'asymptotic simplicity' in the class of all ('reasonably' defined) asymptotically flat solutions.

\subsection{The regular finite Cauchy problem}

Under the conformal embedding of Minkowski space into the Einstein cosmos described above, the Cauchy hypersurface $\tilde{S}=\{t=0\} \simeq \mathbb{R}^{3}$ with its interior Euclidean metric $\tilde{e}$ is mapped conformally into the unit 3 -sphere, $\tilde{S} \rightarrow S \equiv\{s=$ $0\}=\tilde{S} \cup\{i\}$, with its standard metric $d \omega^{2}$ and a point $i$ with coordinates $s=0$, $\chi=\pi$ which represents space-like infinity for $(\tilde{S}, \tilde{e})$. If more general data $\tilde{h}, \tilde{\chi}$ satisfying the vacuum constraints are prescribed on $\tilde{S}$ which are asymptotically Euclidean in the sense that $\tilde{h}$ suitably approaches the metric $\tilde{e}$ near spacelike infinity, it appears reasonable to conformally compactify $(\tilde{S}, \tilde{h})$ by suitably rescaling $\tilde{h} \rightarrow h=\Omega^{2} \tilde{h}$ and adding a point to $\tilde{S}$ as above. Depending on $\tilde{h}$, there may not exist coordinates $x^{\alpha}$ on $S$ near $i$ and a conformal factor $\Omega$ such that the metric coefficients $h_{\alpha \beta}$ will be smooth at $i$. But even if the data $\tilde{h}, \tilde{\chi}$ are chosen such that $h$ extends smoothly to $i$, in fact, even if $\tilde{\chi}=0$, it turns out that in coordinates adapted to $h$ the rescaled conformal Weyl tensor behaves as

$$
d^{\mu}{ }_{\nu \lambda \rho}=O\left(r^{-3}\right) \quad \text { as } \quad r \rightarrow 0,
$$

where $r$ denotes the $h$-distance from $i$, unless the ADM mass of the data vanishes, i.e. $\tilde{h}$ is flat.

To let things not look utterly hopeless, we note that $d^{\mu}{ }_{\nu \lambda \rho}$ is not an arbitrary tensor with Weyl symmetry satisfying (52), that its structure near $i$ is restricted by the assumption that $\tilde{h}$ and $\tilde{\chi}$ be asymptotically Euclidean, satisfy the constraints, and that $d^{\mu}{ }_{\nu \lambda \rho}$ is given by a specific formula. We refer to [38] for explicit general expressions.

In the case of Minkowski space the hypersurfaces $\mathcal{J}^{ \pm}$, which are swept out by the future resp. past directed null geodesics emanating from the point $i^{0}$ (naturally identified with $i \in S$ ), are characteristics for the conformal field equations in the conformally extended space-time. If we assume for a moment that a similar picture can be established for the space-time which develops from 
non-trivial data $\tilde{h}, \tilde{\chi}$, then (52) makes us wonder why this strong singularity at $i$ does not spread along the characteristics $\mathcal{J}^{ \pm}$and destroy the smoothness of the conformal structure there in the first place! If such a contradiction does not arise, it can only be due to (a) specific features of the field equations and/or (b) specific requirements on the data which are analogous to the regularity conditions observed in the hyperboloidal initial value problem.

If features as referred to in (a) exist, they must lie beyond conformal regularity. If requirements such as those referred to in (b) exist we may wonder what kind of conditions could, in a situation where the conformal extension is only given by a point, replace the vanishing of certain functions on a 2 -surface as required in the hyperboloidal problem. Moreover, if one tries to analyze an initial value problem for the conformal field equations for data as singular as (52) the choice of a gauge becomes a delicate problem and there is little lee-way for rough estimates.

Somewhat unexpectedly it turns out that for suitably chosen asymptotically flat initial data the standard Cauchy problem can be reformulated as a regular finite initial value problem for the general conformal field equations with data given on a compact manifold with boundary. Moreover, the underlying setting also allows us to analyze to some extent the questions raised above.

The underdetermined quasi-linear system of constraints imposes very weak conditions on the data. In particular, it requires very little with respect to the smoothness of the data at space-like infinity. Thus it is clear that we have to make assumptions concerning the precise behaviour of the data near $i$. In [38], were the setting has been developed and worked out in detail as described below, it was assumed that the data are time-symmetric, that $h$ extends smoothly to $i$, and that $h$ is in fact analytic in normal coordinates at $i$. It follows in particular that $\tilde{\chi}=0$ and that the conformal factor $\Omega$ which relates $h$ to the physical metric $\tilde{h}=\Omega^{-2} h$ satisfies $\Omega \in C^{2}(\bar{S}) \cup C^{\infty}(\bar{S} \backslash\{i\})$ with $\Omega=0, d \Omega=0$, Hess $\Omega \simeq h$ at $i$, but $\Omega$ is not smooth at $i$ unless the data have vanishing ADM mass. As a consequence we still have (52). It should be emphasized that our assumptions have been made for convenience because the structure of the data near space-like infinity needs to be known in all details (cf. the remarks below). In particular, the assumption of analyticity can be dropped and replaced by a $C^{\infty}$ condition.

\subsubsection{The cylinder at space-like infinity}

In the following we shall describe the main considerations leading to the formulation of the regular finite initial value problem near space-like infinity. The general conformal field equations will be used here for several reasons. We want to obtain a formulation which is leading to statements which depend as far as possible on the conformal structure itself and as little as possible on conditions 'put in by hand'. The nature of the singularity at $i$ forces us to carefully distinguish between the dependence of the fields on radial and angular directions at $i$. The frame field in the formalism is readily adapted to this situation. Much more importantly, we want to avoid any gauge which depends on implicit 
wave equations, because these might transport non-intrinsic singularities along null infinity. An example for this occurs if the conformal gauge in the metric conformal field equations is removed by prescribing the Ricci scalar. Clearly, space-like infinity is not met by time-like conformal geodesics starting at points of $\tilde{S}=S \backslash\{i\}$ and conformal Gauss systems should thus be unaffected by the singularity at $i$. Finally, the location of the set where the conformal factor vanishes will be known a priori.

In 38] the general conformal field equations have been used in the spin frame formalism, because this leads to various algebraic simplifications. Here we will discuss the standard frame formalism, suppressing many details for which we refer the reader to 38 .

Assume a fixed choice of the conformal scaling for the initial data. Choose a fixed oriented $h$-orthonormal frame $e_{a}, a=1,2,3$ at $i$. Any other such frames at $i$ is then obtained in the form $e_{a}(s)=s^{c}{ }_{a} e_{c}$ with $s=\left(s^{c}{ }_{a}\right) \in S O(3)$. For given $s$, we distinguish $e_{3}(s)$ as the radial vector at $i$ and assume $e_{a}(s)$ to be parallelly transported with the Levi-Civita connection of $h$ along the geodesics with tangent vector $e_{3}(s)$ at $i$. We denote by $\rho$ the affine parameter on these geodesics which vanishes at $i$ and denote the frame obtained from $e_{a}(s)$ at the value $\rho$ by $e_{a}(\rho, s)$. It will be assumed that $|\rho|<a$, where $a$ is chosen such that the metric ball $B$ centered at $i$ with radius $a$ is a convex normal neighbourhood for $h$. Then $]-a, a\left[\times S O(3) \ni(\rho, s) \rightarrow e_{a}(\rho, s) \in S O(S)\right.$ defines a smooth embedding of a 4-dimensional manifold into the bundle $S O(S)$ of oriented orthonormal frames over $S$.

We denote by $\hat{B}$ the image of the set $\left[0, a\left[\times S O(3)\right.\right.$, by $I^{0}$ its boundary $\{\rho=0\} \simeq S O(3)$, and by $\pi$ the restriction to $\hat{B}$ of the projection of $S O(S)$ onto $S$. For given $s \in S O(3)$ the vectors $e_{3}\left(s s^{\prime}\right)$ are identical for $s^{\prime} \in S O(2)$, the subgroup of $s=\left(s^{c}{ }_{a}\right) \in S O(3)$ for which $s^{c}{ }_{3} e_{c}=e_{3}$, and all these vectors are parallelly transported along the same geodesic. It follows that the set $\hat{B}$ inherits from $S O(S)$ an action of the subgroup $S O(2)$ which implies a factorization $\hat{B} \stackrel{\pi^{\prime}}{\rightarrow} B^{\prime}=\hat{B} / S O(2) \stackrel{\pi^{\prime \prime}}{\rightarrow} B$ of the map $\pi$ such that $\pi^{\prime \prime}$ maps the set $\pi^{\prime}\left(I^{0}\right) \simeq S^{2}$ onto $i$ while it implies a diffeomorphism of $B^{\prime} \backslash \pi^{\prime}\left(I^{0}\right)$ onto the punctured ball $B \backslash\{i\}$ which we could use to identify these sets.

It turns out more convenient, however, to pull the initial data on $B$ via $\pi$ back to $\hat{B}$, use this set as the initial manifold and $\rho$ and $s$ as 'coordinates' on it. Questions of smoothness are then easily discussed and certain expressions simplify considerably. Since all fields have a well defined transformation behaviour (spin weight) under the action of $S O(2)$ and this action commutes with the evolution defined by the propagation equations, no problem will arise from this. In 38] it is shown how to prescribe on the part of $\hat{B}$ where $\rho>0$ a frame field $X, c_{a}(\rho, s), a=1,2,3$, where $X$ is generated by the action of $S O(2)$, the vector fields $c_{a}(\rho, s)$ satisfy $T(\pi) c_{a}(\rho, s)=e_{a}(\rho, s)$, and a frame formalism can be applied. In the coordinates $\rho$ and $s$ on $\hat{B}$, which are essentially spherical coordinates, some frame vector fields and certain data derived from them are singular at $\rho=0$.

With a suitable choice of the initial data for the conformal geodesics the 
conformal factor and the 1-form $d_{k}$ take the form

$$
\Theta=\frac{\Omega}{\kappa_{*}}\left(1-\tau^{2} \frac{\kappa_{*}^{2}}{\omega_{*}^{2}}\right), \quad d_{0}=-2 \tau \frac{\kappa_{*} \Omega_{*}}{\omega_{*}^{2}}, \quad d_{a}=\frac{1}{\kappa_{*}}\left(e_{a}(\Omega)\right)_{*}
$$

where $\omega^{2}=2 \Omega\left|h^{\alpha \beta} \Omega_{, \alpha} \Omega_{, \beta}\right|^{-1 / 2}$ and $\kappa$ is a function on $\hat{B}$ which we are free to choose. Any function $f_{*}$ in (53) is considered to be constant along the conformal geodesics and to agree with its given initial value $f$. In the conformal extension of Minkowski space in which space-like infinity is represented by the point $i^{0}$ we would have $\kappa=O(1)$ as $\rho \rightarrow 0$. For our purpose it will be much more useful to choose $\kappa$ in the form $\kappa=\rho \kappa^{\prime}$ with some smooth scalar (i.e. $S O(2)$-invariant) function $\kappa^{\prime}$ on $\hat{B}$ with $\kappa^{\prime}=1$ on $I^{0}$ such that $\left.\Theta\right|_{\tau=0}=\frac{\Omega}{\kappa_{*}}=O(\rho)$ as $\rho \rightarrow 0$. In this particular conformal gauge all data extend smoothly to $I^{0} \subset \hat{B}$ and the coefficient functions $\Theta, d_{k}$ in the general conformal field equations are smooth as well.

We are free to choose $\kappa^{\prime}$. For definiteness we set $\kappa=\omega$ such that $\Theta=0$ for $\tau= \pm 1$. If the resulting coordinates remain regular long enough, future and past null infinity will be represented near space-like infinity by the sets $\mathcal{J}^{ \pm}=\{\tau= \pm 1\}$, while space-like infinity will now be represented by the cylinder at space-like infinity which is given by the set $I=\{\rho=0,|\tau|<1\}$. It 'touches' null infinity at the sets $I^{ \pm}=\{\rho=0, \tau= \pm 1\}$. What in the beginning was a Cauchy problem now looks like an initial boundary value problem. It turns out, however, that the boundary $I$ is of a very special nature.

If we write $w=\left(e^{\mu}{ }_{k}, \hat{\Gamma}_{i}{ }^{j}{ }_{k}, \hat{L}_{j k}\right)$ and the unknown (38) in the form $u=(w, z)$ with $z=\left(d^{i}{ }_{j k l}\right)$, the symmetric hyperbolic reduced equations take the form

$$
A^{\mu}(w) \partial_{\mu} z=H(w) z, \quad \partial_{\tau} w=F(w, z),
$$

(where we suppress the dependence of $F$ on the conformal factor $\Theta$ and the 1form $d_{k}$ ). Extending the data on $\hat{B}$ as well as the evolution equations (preserving symmetric hyperbolicity) smoothly into the domain $\rho<0$, standard results imply the existence of a smooth local solution in a neighbourhood of the initial hypersurface which contains a piece of $I$. The fact that $\Theta=0$ on $I$ then allows us to conclude from the details of the equations above that

$$
A^{\rho}=0 \quad \text { on } \quad I \text {. }
$$

This implies that $I$ is a total characteristic in the sense that the complete system (54) reduces on $I$ to a symmetric hyperbolic system of inner equations on $I$. It follows that $u$ is determined uniquely by its values on $I^{0}$, that the solution obtained for $\rho \geq 0$ does not depend on the chosen extension of the data through $I^{0}$, and that $u$ extends smoothly to $I$ (which can not be taken for granted in general initial boundary value problems, cf. [35], [44]).

The construction of $I$ can be considered as attaching a boundary at spacelike infinity to the physical space-time. However, we wish to emphasize that not the definition of a boundary but the analysis of the field equations is our main interest. We do not impose any conditions on the time evolution of the 
solutions except the field equations. The construction is defined in terms of general properties of conformal geometry, the conformal field equations, and the choice of the initial data for the gauge.

While everything extends smoothly to $I$ near $I^{0}$, it should be observed that $I$ is not a geometrical entity but that it is attained as a certain limit of geometric objects, namely the conformal geodesics defining our gauge. It is not a timelike hypersurface and some of the metric coefficients do in fact diverge at $I$ in the given coordinates. This is consistent with the smoothness of the frame coefficients and the vanishing of the matrix $A^{\rho}$ on $I$. Since the wave equation for the rescaled conformal Weyl tensor would degenerate at $I$, it turns out important here that we extract the hyperbolic system from the first order Bianchi equation. It is remarkable that the blow-up procedure $i^{0} \rightarrow I$, the coordinate $\rho$ which is adapted to the conformal scaling defined on $S$ by $\Omega$, and the conformal gauge defined by $\Theta$ conspire to produce a regular finite problem. By a simple rescaling (different choice of $\kappa$ ) and a corresponding redefinition of the coordinates the representation of space-like infinity in terms of the cylinder $I$ can be converted into the conventional representation of space-like infinity by a point $i^{0}$ (38]).

\subsubsection{The transport equations on the cylinder at space-like infinity}

The procedure described above, by which the point $i^{0}$ is replaced by the cylinder $I$, does not only give us regular data and equations but also leads to an unfolding of the evolution process near space-like infinity which allows us to analyze the process there at arbitrary order and in all details. Taking formal derivatives of the evolution equations with respect to $\rho$, restricting to the cylinder, and observing (55), one obtains transport equations on $I$ for all the functions $u^{p}=$ $\left.\partial_{\rho}^{p} u\right|_{I}, p=0,1, \ldots$, on the cylinder. Expanding $u^{p}=u^{p}(\tau, s)$ in terms of a certain functions system $T_{2 m}{ }^{i}{ }_{k}$ (where $m=0,1,2, \ldots$ and $i, k=0, \ldots, 2 m$ ), on $S O(3)$ (resp. on $S U(2)$ in the case of the spin frame formalism used in [38]), these equations reduce to a hierarchy of ODEs along the curves $]-1,1[\ni \tau \rightarrow$ $(\tau, \rho=0, s) \in I$, where the differential operator depends on $p$ but not on the data while the right hand side of the equation for $u^{p+1}$ depends quadratically on $u^{0}, \ldots, u^{p}$ and on the coordinates.

The calculation of $u$ on $I$ allows us to determine $A^{\mu}(u)$ on $I$, which gives in particular

$$
A^{\tau}=\operatorname{diag}(1+\tau, 1,1,1,1-\tau) \quad \text { on } \quad I .
$$

While the second of equations (54) is an ODE which is regular at $I^{ \pm}$, the matrix $A^{\tau}$ occurring in the first of equations (54) degenerates precisely on $I^{ \pm}$. All the remaining open questions concerning the regular finite initial value problem near space-like infinity are related to this fact.

This degeneracy (which is removed at the next order in $\rho$ ) has important consequences. It turns out that in general the quantities $u^{p}$, in particular the expansion coefficients of the rescaled conformal Weyl tensor, contain terms which behave as $(1-\tau)^{k} \log ^{j}(1-\tau)$ at $I^{+}$. In the expressions which so far have been calculated explicitly only terms with the powers $j=0,1$ occur, but due to the 
non-linearity of the equations other powers should arise as well. Because of the hyperbolicity of the reduced equations these singularities can be expected to spread along $\mathcal{J}^{ \pm}$. The powers $k$ and $j$ observed in the explicit calculations then suggest that in general not all components of $d^{i}{ }_{j l m}$ will be bounded at null infinity. In fact, in the spinor notation used in (47), (48) it appears that in general the rescaled Weyl spinor field will have an unbounded component $\phi_{\text {abcd }} \iota^{a} \iota^{b} \iota^{c} \iota^{d}$ which behaves as $\log (1-\tau)$ at $\mathcal{J}^{+}$, where $\tau \rightarrow 1$.

This suggests that in general the conformal Weyl tensor will vanish on $\mathcal{J}^{+}$ but will neither be differentiable there nor satisfy the assumption (48).

Some care is required here, because the hyperbolicity does not extend to $I^{ \pm}$. However, if the whole setting developed in 38] is linearized at Minkowski space, a complete analysis of the situation can be given which confirms the conclusion (cf. J. Valiente-Kroon, this volume).

Due to the geometric nature and the conformal invariance of our gauge conditions we are discussing here intrinsic features of the solutions. If the null infinities of the solutions would admit smooth extensions of the conformal structures, then the conformal geodesics, the conformal factor, and the frame would extend smoothly through null infinity. Also the components of the rescaled conformal Weyl tensor in that frame would then extend as smooth functions of $\tau$ through null infinity. Thus the observations above suggest that even under assumptions on the conformal data which cannot be improved from the point of view of smoothness there will in general occur logarithmic singularities at null infinity due to the evolution process.

\subsubsection{A conjecture}

The class of logarithmic singularities determined in 38 have been found by solving the transport equations on $I$ explicitly for certain components $u^{\prime p}$ of the functions $u^{p}, p=0,1,2, \ldots$, in the decomposition with respect to the functions $T_{2 m}{ }^{i}{ }_{k}$. Inspecting the initial data $u^{\prime p}$ for these quantities on $I^{0}$ and relating them to the free datum $h$ on $\tilde{S}$, we find that the logarithmic terms we find for general data in our class do not occur in $u^{\prime p}$ for $p \leq q_{*}+2$ if and only if $h$ satisfies the regularity condition

$$
\mathcal{S}\left(D_{i_{1}}, \ldots, D_{i_{q}} B_{j k}(i)\right)=0 \quad \text { for } \quad q=0,1, \ldots, q_{*},
$$

where $D$ denotes the connection and $B_{j k}=\frac{1}{2} \epsilon_{j}{ }^{i l} D_{i} L_{l k}$ the Cotton tensor of $h$ while $\mathcal{S}$ denotes the operation of taking the symmetric trace-free part of a tensor.

Consistent with the fact that the free initial datum is given by the conformal structure of $h$, condition (56) is conformally invariant for given $q_{*} \geq 0$. The condition has been found earlier in the quite different, though related, context of vanishing ADM mass ([31]). Although the derivation is much easier in that case and related to a geometric picture, we are still lacking a geometrical interpretation of condition (56).

Since the condition is conformally invariant and it is easy to construct data which are conformally flat near space-like infinity, there exist many non-trivial 
data satisfying the condition. With the techniques of 15 and [16] such data can even be constructed so that they agree with prescribed data on a compact set. Moreover, there exists a large class of data which satisfy the condition in a non-trivial way (38). In fact, all data which are conformal to static data near space-like infinity satisfy (56) for all $q_{*}$ (31]). Since static data are analytic near space-like infinity $([6],[59])$, it follows that condition (56) can be satisfied with Cotton tensors which do not vanish near space-like infinity (38). This is consistent with the fact that the static solutions represent the largest class of time-symmetric solutions which are known to admit near space-like infinity a smooth structure at null infinity. It would be interesting to know whether the results of [15] and [16] can be generalized to glue static ends to given data.

For various reasons we expect condition (56) to be of a much wider significance.

Conjecture: There exists an integer $k_{*} \geq 0$ such that for given $k \geq k_{*}$ the time evolution of an asymptotically Euclidean, time-symmetric, conformally smooth initial data set admits a conformal extension to null infinity of class $C^{k}$ near space-like infinity, if the Cotton tensor satisfies condition (56) for a certain integer $q_{*}=q_{*}(k)$.

If this conjecture can be shown to be correct, solutions for data which satisfy (56) for all $q_{*}$ will contain smooth hyperboloidal hypersurfaces. We expect that the corresponding hyperboloidal initial data approach Minkowskian hyperboloidal data in a continuous way if the Cauchy data approach Minkowski data. This then can be used in conjunction with the results of 30 about the hyperboloidal Cauchy problem to show the existence of solutions satisfying all the requirements of definition (3.1). Because of the degeneracy of the equations at $I^{ \pm}$the proof of the conjecture is not a standard problem and requires new ideas. However, we can split off an important and interesting subproblem.

Subconjecture: If condition (56) holds for a given $q_{*} \geq 0$, the functions $u^{p}, p \leq q_{*}+2$, extend smoothly to $I^{ \pm}$.

In the formalism employed in 38 the validity of the statement above can in principle be checked by a recursive calculation of the functions $u^{p}$. In fact, such a calculation shows that the $u^{p}$ are smooth at $I^{ \pm}$for $p \leq 3$ if the corresponding regularity conditions hold (38, [42]). The general proof essentially reduces to controlling the algebraic structure of the terms on the right hand side of the ODEs governing the transport of the $u^{p}$, which, with increasing order, becomes quite complicated (cf. the discussion in [42, 443]). It is most desirable to determine terms of higher order by an explicit calculation, possibly with an algebraic computer program, to obtain an insight into the general structure of the functions $u^{p}$. This will help with a general analytic proof of the subconjecture and also provide indispensable information for solving the regular finite initial value problem numerically.

\subsubsection{Problems and prospects}

Condition (56) suggests that data for solutions satisfying the requirements of definition (3.1) have to satisfy at $i$ conditions at all orders. Therefore, a gener- 
alization of the regularity condition to the case of data with non-trivial extrinsic curvature requires a general and very detailed investigation of the solutions to the constraints near space-like infinity. Such a study has recently been carried out (cf. S. Dain, this volume) and the generalization of the analysis of [38] to a much larger class of data, which also requires new considerations concerning the gauge, is possible.

The proof of our conjecture, or its generalization to the case of non-trivial extrinsic curvature, requires to show the existence of solutions to the finite regular initial value problem near $I$ whose underlying manifolds contain a piece of the form $\left\{|\tau|<1,0 \leq \rho<\rho_{0}\right\}$. Moreover, we need to obtain sufficient control on the smoothness of the solutions near $\mathcal{J}_{\rho_{0}}^{ \pm}=\left\{|\tau|=1,0 \leq \rho<\rho_{0}\right\}$. The specification of the precise values of $k_{*}$ and $q_{*}(k)$, possibly referring to Sobolev spaces, should be part of this proof. In principle it is not only of interest to see whether the conjecture can be justified but we would also like to know how the logarithmic terms propagate along $\mathcal{J}_{\rho_{0}}^{+}$, if they do so, and what the role and meaning of these terms is.

It would also be desirable to sort out for more general classes of free data the type of non-regularities which arise from solving the constraints and from solving the evolution equations. Since free data can be quite 'rough' at spacelike infinity while still being asymptotically Euclidean, there has to be made a reasonable choice. There is no point in striving for a generality which is not required and of no use in modeling physical systems. However, already for quite natural looking free data the constraints can imply terms of the form $\rho^{k} \log ^{j} \rho$ on $\tilde{S}$ (cf. S. Dain, this volume). Clearly it is of interest to see to what extent our analysis can be generalized to handle such data even if our conjecture and its possible generalizations to more general data bear out. It will be the only way to assess the generality and the role of the solutions which admit a smooth structure at null infinity in the class of all solutions arising from asymptotically Euclidean data.

If the smoothness of the conformal structure obtained at null infinity turns out to be much weaker than expected in systems which appear of "physical importance, we will have to reconsider definition 3.1. While it may not be applicable any longer in the desired generality, the underlying idea is viable because the conformal field equations may be used to control also solutions with a 'rough' asymptotic behaviour ([14, [60], cf. also P. T. Chruściel, this volume). If the solutions for suitable data admit polyhomogeneous expansions in $\tau$, we can expect to relate the coefficients of this expansion, which can be considered as functions on $\mathcal{J}^{+}$, again to the initial data. This should allow us to give some interpretation for these terms.

In all such studies the insight into the equations gained by trying to establish the extremely sharp characterization of the fall-off behaviour of gravitational fields in terms of asymptotic simplicity will prove important. For instance, the general analytic proof of the subconjecture stated above will be of particular

\footnotetext{
${ }^{1}$ Besides getting control on the mathematical possibilities, finding out which data are of 'physical importance' may turn out to be the most interesting part of the whole exercise. The interpretation of initial data is still a wide open field.
} 
interest because it will provide a further understanding of the peculiar features of the field equations. The precise structure of the lower order terms in the equations, which is essentially determined by the fact that we are dealing with a soldered gauge theory (cf. the discussion of the general conformal field equations in the context of normal conformal Cartan connections in [35]), becomes critical here. Understanding its role in the context of the data dependent conformal singularity at space-like infinity may shed light also on the mechanism of the field equations in other singular situations.

The concept of an isolated system is not part of the general theory but a matter of expediency (cf. the discussion in [36]). The final criterion for its definition is the possibility to model physical systems in a way which allows us to extract interesting information about physical processes. If the conjecture above bears out, the resulting space-times will allow us to perform very detailed discussions of all their physical aspects and the proof of the conjecture will provide information which reduces many discussions to direct calculations.

An example for this is given by the calculation of the NP-constants in terms of the initial data in [42. As neatly discussed in [20], these provide interesting restrictions on the global structure of solutions. Another example is the decision on the reducibility of the BMS group to the Poincaré group in 42]. So far both results still rely on the correctness of our conjecture. In fact, many earlier studies of physical concepts associated with the asymptotic structure will be put on a firm analytical footing.

If logarithmic singularities at null infinity (or the coefficients of the logarithmic terms) turn out to be of any physical significance this will not only create problems for the concept of asymptotic simplicity but also for the conventional method of calculating gravitational radiation by solving numerically initial boundary value problems for Einstein's field equations. In that approach the singularities and what might be lost by ignoring them cannot even be seen. While the initial data for the initial boundary value problem can be arranged not to be affected by the modification of the data near space-like infinity, with our present knowledge there appears to be no way, whatever boundary treatment in the numerical calculation is used, to control whether one is calculating a solution where the influence of such a modification enters through the boundary into the numerical solution (cf. 44 for the amount of freedom and control available in the analytic treatment of the initial boundary value problem for Einstein's field equations).

On the other hand if the latter method makes any sense at all, it may for practical purposes be quite sufficient and of advantage to calculate numerically asymptotically simple solutions for data with a Schwarzschild end if the procedures in [15], 16] are amenable to a numerical treatment. It is quite simple to identify in the evolution of such data hyperboloidal hypersurfaces which intersect $\mathcal{J}^{+}$in the Schwarzschild part of the evolution and which extend with the implied data smoothly as solutions to the conformal constraints across $\mathcal{J}^{+}$ (41]). In fact, the corresponding hyperboloidal data for the conformal field equations can be calculated by solving a system of ODEs ([38]). This would resolve a number of difficult problems occurring in the present numerical codes 
(cf. J. Frauendiener and S. Husa, this volume).

Again, while this in the end may turn out sufficient for practical purposes, we have no possibility to decide on that before the existence of asymptotically simple solutions has been discussed under sufficiently general assumptions. In that case quite general complete maximal hyperbolic solutions together with their asymptotics may become calculable from standard Cauchy data and the interpretational framework associated with the concept of asymptotic simplicity may allow us to draw the desired conclusion.

It appears that presently the conformal field equations offer the only way to perform such calculations. In the case of the characteristic method, which also allows one to calculate numerically semi-global solutions, there may arise problems because null hypersurfaces have the tendency to develop caustics (an intrinsic feature which cannot be overcome by smoothing procedures, cf. [47] for a discussion of caustics in the context of the characteristic initial value problem and G. Galloway, this volume, for an illustration of the ubiquity of conjugate points on null geodesics). There are also problems in prescribing appropriate initial data. Assuming past time-like infinity to be represented by a regular point, one could think of prescribing null data on the set $\mathcal{J}^{-} \cup\left\{i^{-}\right\}$which only has a very special and well understood 'caustic' at $i^{-}$. However, at present it is by no means clear how to prescribe these data such that they develop into a solution which admits an asymptotically flat Cauchy hypersurface. Similar difficulties occur if one tries to invent other ways of calculating complete spacetimes from characteristic data.

\subsection{Time-like infinity}

It is a remarkable feature of the conformal Einstein equations, which should not be taken for granted, that they force the null generators of $\mathcal{J}^{+}$to meet in precisely one regular point $i^{+}$of the conformal extension if the solution exists for long enough in a sufficiently regular fashion and the initial data are prescribed appropriately ([30]). In such a situation 'time-like infinity' has a clear-cut meaning, it is locally similar to that of Minkowski space, and it can 'readily' be attained in numerical calculations ([54], cf. also S. Husa, this volume). The calculation of $i^{+}$provides an opportunity to test numerical codes in

long range calculations of space-times and to perform studies of the non-linear interaction of gravitational waves under well controlled conditions, however, it is of course not a purpose in itself.

To calculate the gravitational radiation emitted by the merger of two black holes we will have to consider solution which develop singularities and horizons. While it may appear wise in a numerical calculation to avoid, if possible, entering the horizon and hitting the singularity, such an attitude does not help in an analytical investigation and we do, in fact, consider it necessary to attack the situation head on. In fluid mechanics there exists a long tradition of studying singularities (shocks) and there have been developed methods to handle them in numerical calculations. The singularities observed in general relativity, however, are in general much more complicated and severe from a conceptional point of 
view as well as from the point of view of actual analytical expressions. In spite of a considerable effort to come to grips with them analytically and, more recently, numerically (cf. D. Garfinkle, this volume, and also [7] and the literature given there, which discusses some remarkable achievements), we are still far from a situation where we could attain the desired information in sufficiently general situations. In fact, the 'well-known' Schwarzschild-Kruskal or Kerr solutions still pose difficulties.

There exist several ways to discuss 'singular points of space-times' in an abstract way (cf. [71 and the references given there), however, there are no concepts available which would allow us to perform under general assumptions an analysis of singularities in the context of the field equations. While it is a central problem in singular situations to obtain detailed statements which are independent of any specific gauge, it is at least as difficult to develop gauge conditions which allow us to arrive at any interesting statement at all. The latter problem certainly does not have an universal solution. The best we can hope for is to find manageable conditions which by their general nature and by experience with specific solutions may be expected to work for certain classes of solutions.

We have seen above that conformal Gauss systems have many nice local properties and the discussion of the regular finite Cauchy problem suggests that they also behave nicely near space-like infinity. It can also be shown that they can be used in calculations of $i^{+}$from hyperboloidal Cauchy data which are sufficiently close to Minkowskian hyperboloidal data. However, in all these situations the gravitational field must be considered as weak and one would like to know whether conformal Gauss systems can also exist globally and with good asymptotic properties on asymptotically flat space-times which posses regions of strong curvature or even singularities and horizons.

Caustics of congruences of metric geodesics arise because the latter obey equations of second order. Congruences of conformal geodesics are governed by equations of third order (cf. 64 ) and are thus even more prone to caustic formation, in fact, different conformal geodesics may become tangent to each other. However, the same structural property which may be responsible for such severe caustics allows us to show that there exist conformal Gauss systems on the Schwarzschild-Kruskal solution which smoothly cover the entire spacetime and extend smoothly and without degeneracy through $\mathcal{J}^{ \pm}$into any smooth extension of the space-time through null infinity (41).

These systems are characterized by initial data for the conformal geodesics on the hypersurface $\tilde{S}$ of the Schwarzschild-Kruskal solution which extends analytically the hypersurface $\{t=0\}$ of the Schwarzschild space. The data considered in [11] are invariant under the space reflection symmetry in $\tilde{S}$ and the rotational symmetry and the congruence is orthogonal to $\tilde{S}$. On $\tilde{S}$ are given the standard spherical coordinates $\phi, \theta$ and the isotropic radial coordinate $r, 0<r<\infty$, which is related to the radial coordinate $\bar{r}$ of the standard Schwarzschild line element $g_{S}=\left(1-\frac{2 m}{\bar{r}}\right) d t^{2}-\left(1-\frac{2 m}{\bar{r}}\right)^{-1} d \bar{r}^{2}-\bar{r}^{2} d \sigma^{2}$ on the subset $\bar{r}<2 m$ by $r=1 / 2(\bar{r}-m+\sqrt{\bar{r}(\bar{r}-2 m)})$. The two asymptotically flat ends of $\tilde{S}$ are 
at $r=0$ and $r=\infty$. The coordinates $\phi, \theta, r$ are dragged along with the congruence and define together with the parameter $\tau$ of the conformal geodesics a smooth coordinate system (ignoring here the singularity inherent in the spherical coordinates which in the present situation can be easily avoided) on the Schwarzschild-Kruskal space with $\tilde{S}=\{\tau=0\}$. The initial data are prescribed such that the conformal factor takes the form $\Theta=\left(r-\frac{m}{2}\right)^{2}\left(r+\frac{m}{2}\right)^{-2}\left(f(r)^{2}-\tau^{2}\right)$ where $f(r) \equiv r /\left(r^{2}-\frac{m^{2}}{4}\right)$.

The coordinates stay regular, because of the specific choice of the 1-form $b$ on $\tilde{S}$. The acceleration of the conformal geodesics, which is determined by $b$, tends by our choice to spread out the curves such that no caustics can develop. This latter fact is established by analyzing an equation satisfied by the connecting vectors of the congruence of conformal geodesics which generalizes the Jacobi equation known for metric geodesics. We consider the fact, that such a geometric analysis is possible as particularly useful.

It is well known that the Schwarzschild-Kruskal solution admits global double null coordinates (cf. B. Schmidt, this volume), however, these coordinates, or coordinates derived from them by simple algebraic relations, do not extend smoothly through $\mathcal{J}^{ \pm}$and they are very specific for the Kerr family. The properties of the conformal Gauss systems mentioned above raise the hope that such systems can be constructed in much more general situations. Of course, while there are a few general considerations which may help here, a good choice of the initial data for the conformal geodesics will depend on the specific solution and one has to gather experience with this, as with any choice of gauge. If conformal Gauss systems are used in the context of the general conformal field equations to perform numerical calculations, the tensor fields entering the generalized Jacobi equations are essentially calculated as one goes along and the possible tendency of the underlying congruence of conformal geodesics to form caustics can be monitored during the calculation.

It should be noted that Gauss systems for which the initial tangent vector field on $\tilde{S}$ and the pull back of the 1-form field $b$ to $\tilde{S}$ are identical, define the same congruence of curves, if these are considered as point sets. The parameterizations of these curves, however, may be different and the systems will thus define different foliations. This freedom has hardly been explored yet.

Since the space reflection in $\tilde{S}$ is given by $r \rightarrow m^{2} / 4 r$, it is sufficient to discuss the conformal geodesics starting at the subset of $\tilde{S}$ where $r \geq m / 2$, which cover a full $\bar{r} \geq 2 m$ part and two 'halves' (one in the past and one in the future of $\tilde{S}$ ) of the $\bar{r}<2 m$ part of the Schwarzschild solution. The conformal geodesics with $\phi=$ const., $\theta=$ const., $r=r_{+} \equiv(3+\sqrt{5}) m / 4$, stay on a hypersurface $\{\bar{r}=5 \mathrm{~m} / 2\}$ of the Schwarzschild-Kruskal space and approach time-like infinity, denoted by $i^{+}$, as $\tau \rightarrow \tau_{i}=2 / \sqrt{5} \mathrm{~m}$. The conformal geodesics starting with $r>r_{+}$run out to null infinity and arrive there as $\tau \rightarrow \tau_{s c r i}=f(r)$, which is consistent with the expression above of the conformal factor.

The curves starting with $\frac{m}{2} \leq r<r_{+}$hit the singularity at $\bar{r}=0$ as $\tau \rightarrow \tau_{\text {sing }} \equiv f(r) H(K(k), k)$, where $k(r) \equiv \sqrt{2}\left(1+\sqrt{m r / 2}\left(r-\frac{m}{2}\right)\right)^{-1 / 2}$, 


$$
\begin{aligned}
& K(k) \equiv \int_{0}^{\pi / 2}\left(1-k^{2} \sin ^{2} x\right)^{-1 / 2} d x, \text { and } \\
& \quad H(x, k) \equiv \tanh \left(\sqrt{\frac{2-k^{2}}{2}} \int_{0}^{x} \frac{d n^{2}(v, k)-\left(1-k^{2}\right)}{d n^{2}(v, k)+\left(1-k^{2}\right)} d v\right),
\end{aligned}
$$

with Jacobi's elliptic function $d n$. They pierce the horizon $\mathcal{H}^{+}=\{\bar{r}=2 m\}$ at $\tau=\tau_{\text {hor }} \equiv f(r) H\left(x_{h}, k\right)$, with $x_{h} \equiv \int_{0}^{\arcsin \left(k^{2} /\left(4-3 k^{2}\right)\right)}\left(1-k^{2} \sin ^{2} x\right)^{-1 / 2} d x$. As to be expected, we find that $\tau_{s c r i} \rightarrow \tau_{i}$ as $r \rightarrow r_{+}$with $r>r_{+}$and also $\tau_{\text {sing }}, \tau_{\text {hor }} \rightarrow \tau_{i}$ as $r \rightarrow r_{+}$with $r<r_{+}$.

Signals periodic in proper time of an observer approaching the horizon are received on $\mathcal{J}^{+}$at Bondi-times which grow only logarithmically?. Consequently, a high resolution is required in a numerical calculation which aspires to probe the field near $i^{+}$by following outgoing null geodesics which start on a Cauchy hypersurface and arrive at null infinity at very late Bondi-times. Therefore we consider it particularly important to acquire a detailed understanding of the characteristic features of the field near $i^{+}$.

It is customary, and natural from the point of view of the causal structure (cf. [71]), to denote time-like infinity in the schematic (conformally compactified) causal diagrams by one or several ideal points $i^{+}$, but the specific structure of the field in the region close to them is little understood. In our representation of the Schwarzschild-Kruskal space the behaviour of the term $(\Theta \bar{r})^{2} d \sigma^{2}$ of the metric $\Theta^{2} g_{S}$ in the limit as $\tau \rightarrow \tau_{i}$ on the hypersurface $\{\bar{r}=\hat{r}\}$ or $\{\bar{r}=$ $2 m$ \} suggests that we should consider $i^{+}$as a point with coordinates $\tau=\tau_{i}$, $r=r_{+}$(the coordinates $\phi$ and $\theta$ loosing their meaning there as at the origin of a polar coordinate system). Without further analysis, however, this can only be considered as a way of speaking. Contrary to what is observed in Minkowski space, $i^{+}$is not only approached by time-like geodesics and by the null generators of $\mathcal{J}^{+}$, but also by null geodesics originating from the interior of the space-time, namely those generating the event horizon and those which stay on the set $\{\bar{r}=3 m\}$ or approach it in the future. Such phenomena do not occur for regular conformal structures and thus $i^{+}$cannot be considered as a point in a suitably defined regular conformal extension.

It is reasonable to expect that conformal geodesics of a regular conformal structure which approach a point $p$ in the underlying manifold $M$ can be extended, perhaps after an admissible reparametrisation, through that point as a smooth solution to the conformal geodesic equation, and that the curve will be time-like at $p$ if it was time-like before (while such a statement is known to hold for metric geodesics it still has to be shown for conformal geodesics). Assuming this to be true, we can observe an effect of the conformal singularity near $i^{+}$ in our global conformal Gauss system. Since $\partial \tau_{s c r i} / \partial r$ converges to a finite negative number as $r \rightarrow r_{+}$with $r>r_{+}$, we see that $\mathcal{J}^{+}$approaches, as to be expected, the hypersurface $\left\{r=r_{+}\right\}$near $i^{+}$under a certain angle. In contrast, we find that $\partial \tau_{\text {sing }} / \partial r \approx\left(r_{+}-r\right)^{-\frac{\sqrt{2}-1}{\sqrt{2}}}$ as $r \rightarrow r_{+}$with $r<r_{+}$, such that the

${ }^{2}$ I owe much to discussions with P. Hübner about this point. However, we both have to acknowledge the priority of W. Allen's formula: eternity is very long, especially near the end. 
graph of $\tau_{\text {sing }}$, and with it also the graph of $\tau_{\text {hor }}$, becomes tangent to $\left\{r=r_{+}\right\}$ at $i^{+}$. As a consequence, the conformal geodesics generating $\left\{r=r_{+}\right\}$become tangent to the null geodesics generating $\mathcal{H}^{+}$when they approach $i^{+}$.

Though this may look like a subtlety, one may wonder about its consequences if one tries to observe the CFL condition in a numerical calculation near $i^{+}$. Moreover, the speed with which the singularity and the horizon approach the hypersurface $\left\{r=r_{+}\right\}$may require special measures to sufficiently resolve these structures numerically.

There are of course much more drastic effects. While the rescaled conformal Weyl tensor (expressed in suitably adapted coordinates or frame fields) extends smoothly to $\mathcal{J}^{+}$, it diverges along the null generators of the horizon like $\Theta^{-1}$ near $i^{+}$, and blows up like $1 / \bar{r}^{3}$ near the singularity (if the conformal factor approaches a positive value there, as it does in our case), resulting in a non-uniformity with strong gradients near $i^{+}$. Moreover, slight changes in the data may lead to a Kerr solution with drastically different behaviour near $i^{+}$, including a Cauchy horizon, a completely different singularity structure, and causality violations (cf. [11]). In recent years considerable work has been done to understand what happens to these structures under perturbations of the data (cf. 56 and the references given there) but these studies supply surprisingly little precise information on the behaviour of the field in the close vicinity of $i^{+}$.

One may object to our discussion that we create unnecessary problems by insisting on conformal rescalings in a region where the field is usually considered as weak. But the phenomena we discuss are present anyway, they are just brought out most succinctly by suitable rescalings. It is not so clear, though, what 'suitable' may mean here. In the case of space-like infinity we have seen that there are representations which allow us to see properties which are hard to analyse with other choices of the gauge.

This raises the question whether there exists a kind of 'resolution of the singularity at time-like infinity' which makes the situation more amenable to a detailed analysis and to a numerical treatment. The situation near $i^{+}$is clearly quite different from that near $i^{0}$. There is nothing near $i^{0}$ which compares with the singularity ' $\{\bar{r}=0\}$ ' and the horizon $\mathcal{H}^{+}$and the way they meet with $\mathcal{J}^{+}$at $i^{+}$. Because of these structures the singularity obtained in a conformal representation which makes time-like infinity a point is much less isotropic than the singularity obtained if space-like infinity is represented by a point. The singularity at $\bar{r}=0$ cannot be removed by a simple rescaling without shifting it to infinity. Moreover, $i^{+}$is causally differently related to the physical spacetime than $i^{0}$. A 'resolution of the singularity at $i^{+}$' must be compatible with a time evolution which essentially takes into account the complete information given by the initial data. It should be emphasized that we are not interested here in a formal definition of a space-time boundary which relies on more or less implicit assumptions of the time evolution of the field. We seek a construction which will help us analyse the latter. This construction can only be fruitful if it follows from the structure of the field equations in a similar way as the cylinder at space-like infinity does.

Looking again at the complicated behaviour of the fields near time-like infin- 
ity suggested in [56], this task may appear hopeless. However, it seems to be the accepted view that in the case of a gravitational collapse to a black hole some kind of universal behaviour can be expected near $i^{+}$in the sense that the field will in general approach near time-like infinity somehow that of a Kerr solution (68]). The recent proof of the Penrose inequality (10], [55]) lends some support to this, because the original argument leading to this inequality relied on the 'establishment viewpoint' indicated above ([69]).

\section{References}

[1] L. Andersson, P.T. Chruściel. On hyperboloidal Cauchy data for the vacuum Einstein equations and obstructions to the smoothness of scri. Comm. Math. Phys. 161 (1994) 533-568.

[2] L. Andersson, P.T. Chruściel. Solutions of the constraint equations in general relativity satisfying hyperboloidal boundary conditions. Dissertationes Mathematicae Polska Akademia Nauk, Inst. Matem., Warszawa, 1996.

[3] L. Andersson, P.T. Chruściel, H. Friedrich. On the regularity of solutions to the Yamabe equation and the existence of smooth hyperboloidal initial data for Einstein's field equations. Comm. Math. Phys. 149 (1992) 587-612.

[4] L. Andersson, G. J. Galloway. DS/CFT and spacetime topology. urlhttp://xxx.lanl.gov/archive/hep-th/0202161

[5] A. Ashtekar. Asymptotic properties of isolated systems: Recent developments. In: B. Bertotti et. al (eds.) General Relativity and Gravitation Dordrecht, Reidel, 1984.

[6] R. Beig, W. Simon. Proof of a multipole conjecture due to Geroch. Comm. Math. Phys. 79 (1981) 581-589.

[7] B. K. Berger. Numerical Approaches to Spacetime Singularities. urlhttp://xxx.lanl.gov/archive/gr-qc/0201056

[8] L. Blanchet. Post-Newtonian Gravitational Radiation. In: B. Schmidt (ed.), Einstein's Field Equations and Their Physical Implications. Berlin, Springer, 2000.

[9] H. Bondi, M. G. J. van der Burg, A. W. K. Metzner. Gravitational waves in general relativity VII. Waves from axi-symmetric isolated systems. Proc. Roy. Soc A 269 (1962) 21-52.

[10] H. L. Bray. Proof of the Riemannian Penrose conjecture using the positive mass theorem. J. Diff. Geom. to appear. urlhttp://xxx.lanl.gov/math.DG/9911173

[11] B. Carter. Global structure of the Kerr family of gravitational fields. Phys. Rev. 174 (1968) 1559-1571. 
[12] Y. Choquet-Bruhat, J. W. York. The Cauchy problem. In: A. Held (ed.) General Relativity and Gravitation, Vol. 1. New York, Plenum, 1980.

[13] Y. Choquet-Bruhat, J. Isenberg, J. W. York. Einstein constraints on asymptotically Euclidean manifolds. Phys. Rev. D 61 (2000) 084034.

[14] P.T. Chruściel, O. Lengard. Solutions of wave equations in the radiation regime. Preprint (2002). urlhttp://xxx.lanl.gov/archive/math.AP/0202015

[15] P.T. Chruściel, E. Delay. Existence of non-trivial, vacuum, asymptotically simple space-times. Preprint (2002). urlhttp://www.phys.univ-tours.fr/ piotr

[16] J. Corvino. Scalar curvature deformation and a gluing construction for the Einstein constraint equations. Comm. Math. Phys. 214 (2000) 137-189.

[17] C. Cutler, R. M. Wald. Existence of radiating Einstein-Maxwell solutions which are $C^{\infty}$ on all of $\mathcal{J}^{-}$and $\mathcal{J}^{+}$. Class. Quantum Grav. 6 (1989) 453-466.

[18] S. Dain. Initial data for stationary space-times near space-like infinity. Class. Quantum Grav. 18 (2001) 4329-4338.

[19] S. Dain, H. Friedrich. Asymptotically Flat Initial Data with Prescribed Regularity. Comm. Math. Phys. 222 (2001) 569-609. urlhttp://xxx.lanl.gov/archive/gr-qc/0102047

[20] S. Dain, J. A. Valiente-Kroon. Conserved quantities in a black hole collision. Class. Quantum Grav. 19 (2002) 811-815.

[21] J. Dieudonné. Treatise on Analysis, Vol IV. Academic Press, New York, 1974.

[22] G. F. R. Ellis, B. G. Schmidt. Classification of singular space-times. GRG 10 (1979) 989-997.

[23] H. Friedrich. On the regular and the asymptotic characteristic initial value problem for Einstein's vacuum field equations. Proceedings of the 3rd Gregynog Relativity Workshop on Gravitational Radiation Theory MPIPEA/Astro 204 (1979) 137-160 and Proc. Roy. Soc., A 375 (1981) 169184.

[24] H. Friedrich. The asymptotic characteristic initial value problem for Einstein's vacuum field equations as an initial value problem for a first-order quasilinear symmetric hyperbolic system. Proc. Roy. Soc., A 378 (1981) 401-421.

[25] H. Friedrich. On the Existence of Analytic Null Asymptotically Flat Solutions of Einstein's Vacuum Field Equations. Proc. Roy. Soc. Lond. A 381 (1982) 361-371. 
[26] H. Friedrich. Cauchy Problems for the Conformal Vacuum Field Equations in General Relativity. Comm. Math. Phys. 91 (1983) 445-472.

[27] H. Friedrich. On the hyperbolicity of Einstein's and other gauge field equations. Comm. Math. Phys. 100 (1985) 525-543.

[28] H. Friedrich. On purely radiative space-times. Comm. Math. Phys. 103 (1986) 35-65.

[29] H. Friedrich. Existence and structure of past asymptotically simple solutions of Einstein's field equations with positive cosmological constant. J. Geom. Phys. 3 (1986) 101-117.

[30] H. Friedrich. On the existence of n-geodesically complete or future complete solutions of Einstein's equations with smooth asymptotic structure. Comm. Math. Phys. 107 (1986) 587-609.

[31] H. Friedrich. On static and radiative space-times. Comm. Math. Phys., 119 (1988) 51-73.

[32] H. Friedrich. On the global existence and the asymptotic behaviour of solutions to the Einstein-Maxwell-Yang-Mills equations. J. Diff. Geom., 34 (1991) 275-345.

[33] H. Friedrich. On the existence of hyperboloidal initial data. In: Z. Perjés (Ed.): Relativity Today. New York: Nova Science, 1992.

[34] H. Friedrich. Asymptotic structure of space-time. In: A.Janis, J.Porter (Eds.): Recent Advances in General Relativity. Boston, Basel, Berlin: Birkhäuser, 1992.

[35] H. Friedrich. Einstein equations and conformal structure: existence of antide Sitter-type space-times. J. Geom. Phys., 17 (1995) 125-184.

[36] H. Friedrich. Einstein equations and conformal structure. In: Geometric Issues in the Foundations of Science P. Tod et al. (eds.), Oxford University Press, Oxford, 1996.

[37] H. Friedrich. Hyperbolic Reductions for Einstein's Equations. Class. Quantum Gravity 13 (1996) 1451-1469.

[38] H. Friedrich. Gravitational fields near space-like and null infinity. J. Geom. Phys. 24 (1998) 83-163.

[39] H. Friedrich. Evolution equations for gravitating ideal fluid bodies in general relativity. Phys. Rev. D 57 (1998) 2317-2322.

[40] H. Friedrich. Einstein's equations and geometric asymptotics. In: N. Dadhich, J. Narlikar (Eds.): Gravitation and Relativity at the Turn of the Millennium. Inter-University Centre for Astronomy and Astrophysics, Pune, India, 1998. urlhttp://xxx.lanl.gov/archive/gr-qc/9804009 
[41] H. Friedrich. Conformal geodesics on vacuum space-times. Preprint 2002. urlhttp://xxx.lanl.gov/archive/gr-qc/0201006

[42] H. Friedrich, J. Kánnár Bondi systems near space-like infinity and the calculation of the NP-constants. J. Math. Phys. 41, (2000), 2195-2232.

[43] H. Friedrich, J. Kánnár. Calculating asymptotic quantities near space-like and null infinity from Cauchy data. Ann. Phys. (Leipzig) 9, (2000), 321330. urlhttp://xxx.lanl.gov/archive/gr-qc/9911103

[44] H. Friedrich, G. Nagy. The initial boundary value problem for Einstein's vacuum field equations. Comm. Math. Phys. 201 (1999) 619-655.

[45] H. Friedrich, A. Rendall. The Cauchy Problem for the Einstein Equations. In: B. Schmidt (ed.), Einstein's Field Equations and Their Physical Implications. Berlin, Springer, 2000.

[46] H. Friedrich, B. G. Schmidt. Conformal geodesics in general relativity. Proc. Roy. Soc. Lond. A 414 (1987) 171-195.

[47] H. Friedrich, J. Stewart. Characteristic Initial Data and Wave Front Singularities in General Relativity. Proc. Roy. Soc. Lond. A 385 (1983) 345-371.

[48] K. O. Friedrichs. Symmetric hyperbolic linear differential equations. Comm. Pure Appl. Math. 7 (1954) 345-392.

[49] R. Geroch. Asymptotic structure of space-time. In: F. P. Esposito, L. Witten (eds.) Asymptotic Structure of Space-Time. New York, Plenum, 1977.

[50] R. P. Geroch, G. Horowitz. Asymptotically simple does not imply asymptotically Minkowskian. Phys. Rev. Lett. 40 (1978) 203-206.

[51] S. W. Hawking, R. Penrose. The singularities of gravitational collapse and cosmology. Proc. Roy. Soc. A 314 (1970) 529-548.

[52] S. W. Hawking, G. F. R. Ellis. The Large Scale Structure of Space-Time. Cambridge, Cambridge University Press, 1973.

[53] P. Hübner. General relativistic scalar field models and asymptotic flatness. Class. Quantum Grav. 12 (1995) 791-808.

[54] P. Hübner. From now to timelike infinity on a finite grid. Class. Quantum Grav. 18 (2001) 1871-1884.

[55] G. Huisken, T. Ilmanen. The Riemannian Penrose inequality. Int. Math. Res. Not. 20 (1997) 1045-1058.

[56] W. Israel. Internal structure of black holes. In: F. W. Hehl. et al. (eds.) Black Holes: Theory and Observation. Berlin, Springer, 1998 
[57] J. Kannar On the existence of $C^{\infty}$ solutions to the asymptotic characteristic initial value problem in general relativity. Proc. Roy. Soc. A 452 (1996) 945-952.

[58] T. Kato. The Cauchy problem for quasi-linear symmetric hyperbolic systems. Arch. Ration. Mech. Anal., 58 (1975) 181-205.

[59] D. Kennefick, N. O'Murchadha. Weakly decaying asymptotically flat static and stationary solutions to the Einstein equations. Class. Quantum. Grav. 12 (1995) 149-158.

[60] O. Lengard. Solution of the Einstein equation, wave maps, and semilinear waves in the radiation regime. $\mathrm{Ph}$. D. thesis, Université de Tours, 2001. urlhttp://www/phys.uni-tours.fr/ piotr/papers/batz

[61] E. T. Newman, R. Penrose. An approach to gravitational radiation by a methods of spin coefficients. J. Math. Phys. 3 (1962) 566-578.

[62] E. T. Newman, R. Penrose. 10 exact gravitationally conserved quantities. Phys. Rev. Lett. 15 (1965) 231-233.

[63] E. T. Newman, R. Penrose. New conservation laws for zero rest-mass fields in asymptotically flat space-times. Proc. Roy. Soc. A 305 (1968) 175-204.

[64] K. Ogiue. Theory of conformal connections. Kodai Math. Sem. Rep. 19 (1967) 193-224.

[65] R. Penrose. Asymptotic properties of fields and space-time. Phys. Rev. Lett., 10 (1963) 66-68.

[66] R. Penrose. Zero rest-mass fields including gravitation: asymptotic behaviour. Proc. Roy. Soc. Lond., A 284 (1965) 159-203.

[67] R. Penrose. Gravitational collapse and space-time singularities. Phys. Rev. Lett., 14 (1965) 57-59.

[68] R. Penrose. Gravitational collapse: the role of general relativity. Riv. Nuovo Cimento, 1 (1965) 252-276.

[69] R. Penrose. Naked singularities. Ann. N. Y. Acad. Sci., 224 (1973) 125134.

[70] R. Penrose. Relativistic symmetry groups. In: A. O. Barut (ed.), Group Theory in Non-Linear Problems. New York, Reidel, 1974.

[71] R. Penrose. Singularities of space-time. In: N. R. Leibowitz et al. (eds.), Theoretical Principles in Astrophysics and Relativity. University of Chicago Press, 1978.

[72] R. Penrose. Singularities and time-asymmetry. In: S. W. Hawking, W. Israel (eds.), General Relativity. Cambridge University Press, 1979. 
[73] R. K. Sachs. Gravitational waves in general relativity VI. The outgoing radiation condition. Proc. Roy. Soc A 264 (1961) 309-338.

[74] R. K. Sachs. Gravitational waves in general relativity VIII. Waves in asymptotically flat space-time. Proc. Roy. Soc A 270 (1962) 103-126.

[75] R. K. Sachs. On the characteristic initial value problem in gravitational theory. J. Math. Phys. 3 (1962) 908-914.

[76] M. Sugiura. Unitary Representations and Harmonic Analysis. North Holland, Amsterdam, 1990.

[77] M. E. Taylor. Partial Differential Equations, Vol. 3. Berlin, Springer, 1996

[78] K. S. Thorne. The theory of gravitational radiation: An introductory review. In: N. Deruelle, T. Piran (eds.) Gravitational Radiation. Amsterdam, North-Holland, 1983.

[79] R. M. Wald. Gravitational collapse and cosmic censorship. In: B. R. Iyer, B. Bhawal, Black Holes, Gravitational Radiation and the Universe. Dordrecht, Kluwer Academic Publishers, 1999.

[80] H. Weyl. Mathematische Analyse des Raumproblems. Springer, Berlin, 1923. Reprinted in: Das Kontinuum. Chelsea Publ. Comp., New York, 1960. 